\title{
DESIGN AND ANALYSIS OF PLATE ANCHORS IN SAND
}

by

Muhammad Waseem

Bachelor of Science Degree in Civil Engineering

University of Engineering and Technology, Lahore, Pakistan, 1995

\author{
A project \\ presented to Ryerson University \\ in partial fulfillment of the requirements for the degree of \\ Master of Engineering in the Program of \\ Civil Engineering
}

Toronto, Ontario, Canada, 2011

(C) Muhammad Waseem 2011 


\section{Author's declaration}

I hereby declare that I am the sole author of this report.

I authorize Ryerson University to lend this thesis to other institutions or individuals for the purpose of scholarly research.

I further authorize Ryerson University to reproduce this thesis by photocopying or by other means, in total or in part, at the request of other institutions or individuals for the purpose of scholarly research. 


\title{
DESIGN AND ANALYSIS OF PLATE ANCHORS IN SAND
}

\author{
Muhammad Waseem \\ Department of Civil Engineering, Ryerson University, Canada, 2011
}

\begin{abstract}
Plate anchors, as an efficient and reliable anchorage system, have been widely used to resist uplift forces produced by structures, such as transmission towers, offshore platforms, submerged pipelines, and tunnels. In order to design a plate anchor it is important to know the factors which influence the design and uplift behavior of anchors embedded in sand.

In this report a number of model uplift tests and numerical investigations made by different authors are described and based on these readings the uplift behavior of anchors in sand is explored and anchor's design procedure is described. In addition, basic anchor types, failure modes in anchors, and design codes are mentioned.

Based on this study, it is found that the failure plane and uplift capacity is significantly influenced by the soil density and embedment depth. Therefore, it is concluded that the influence of sand density and embedment depth should be considered in anchor design.
\end{abstract}




\section{Acknowledgement}

This research could not have been accomplished without the assistance of a number of individuals. I would like to thank my supervisor Dr. Jinyuan Liu for his guidance, advice, and the encouraging comments. In one year of work Dr. Liu always gave insightful opinions that reshaped my thinking and analysis processes. His contribution and the constructive comments in the preparation of this thesis are greatly appreciated.

I am grateful to the Civil Engineering Department at Ryerson University for supporting me and funding for the research work.

Finally I dearly remember my two sons, Ibrahim Waseem and Abdullah Waseem, for their commitment and encouragement during this exercise. 


\section{Dedication}

To my two sons

Abdullah Waseem and Ibrahim Waseem

for their support, encouragement and Love 


\section{Contents}

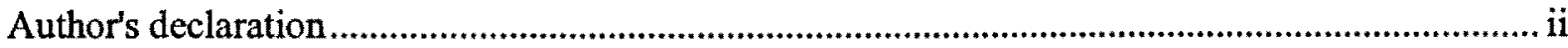

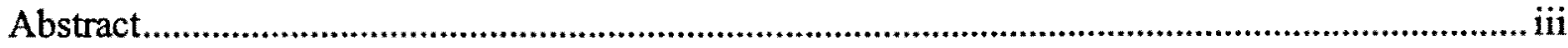

Acknowledgement .................................................................................................................... iv

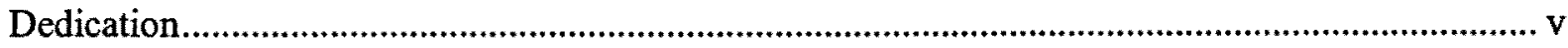

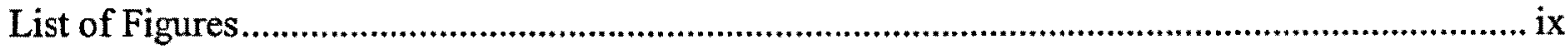

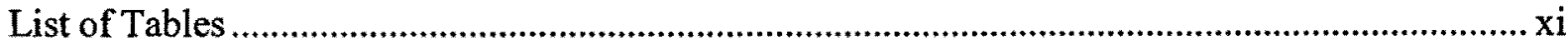

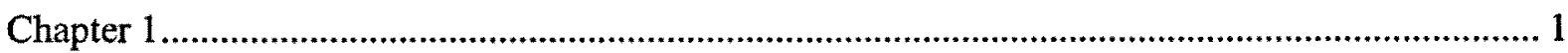

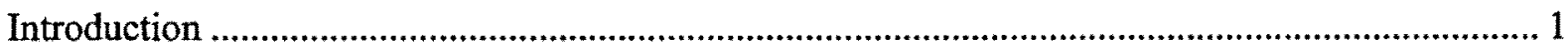

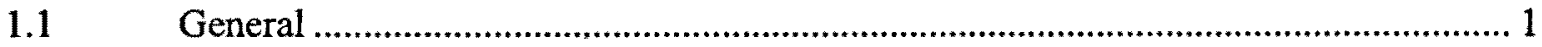

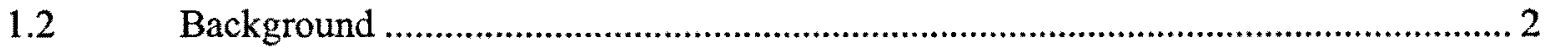

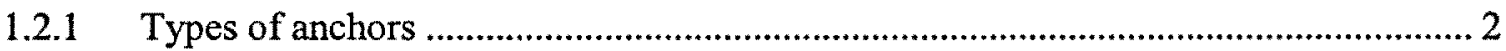

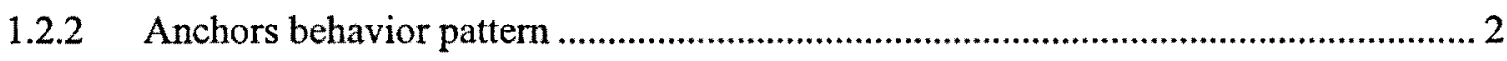

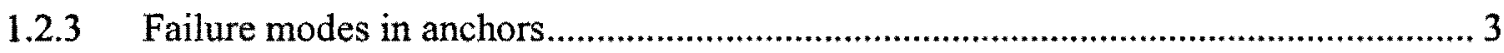

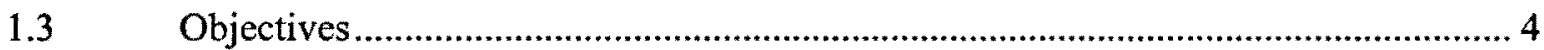

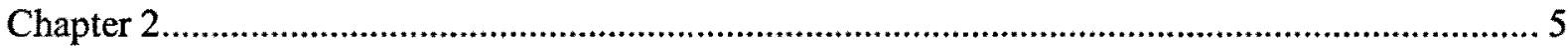

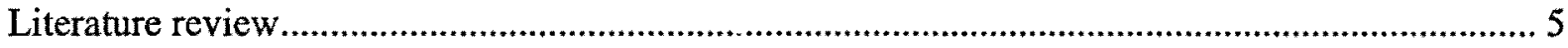

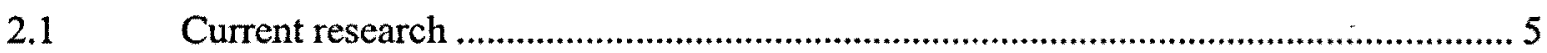

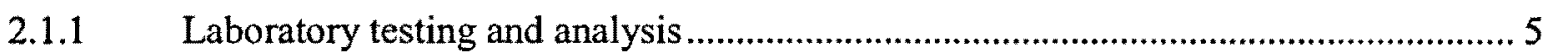

2.1.1.1 Effect of sand grain size, grain shape, sand grading, and density on uplift behavior . 5

2.1.1.2 Delineation of rupture surface in dense sand for shallow anchors............................. 8

2.1.1.3 Delineation of rupture surface in dense sand for deep anchor .................................... 8

2.1.1.4 Relationship between pullout load and displacement for shallow and deep circular

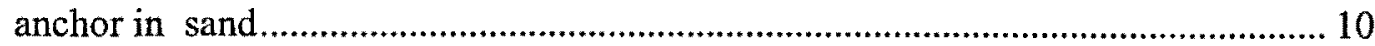


2.1.1.5 Variation of peak pullout load with depth of embedment for circular plate anchors in

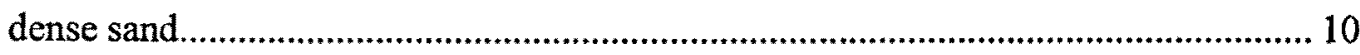

2.1.1.6 Variation of displacement at peak pullout load with embedment ratio....................... 11

2.1.1.7 Variation of peak pullout load with anchor diameter in dense sand ........................... 12

2.1.1.8 Influence of density on peak pullout load for various anchor diameters .................... 14

2.1.1.9 Relationship between breakout factor and embedment ratio for circular plate anchors

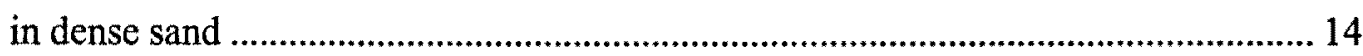

2.1.1.10 Variation of breakout factor with the angle of shearing resistance of sand for various

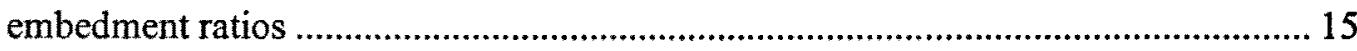

2.1.1.11 Determination of critical embedment ratio, $(\mathrm{H} / \mathrm{D})_{\mathrm{CR}}$, from breakout factor for circular plate anchors in dense sand ........................................................................ 16

2.1.1.12 Ilamparuthi,s proposed empirical design equations .................................................. 18

2.1.1.13 Comparison of measured breakout factor, $\left(\mathrm{N}_{\mathrm{qf}}\right)_{\mathrm{M}}$ and empirically estimated breakout factor, $\left(\mathrm{N}_{\mathrm{gf}}\right) \mathrm{E}$, in medium-dense sand ................................................................... 19

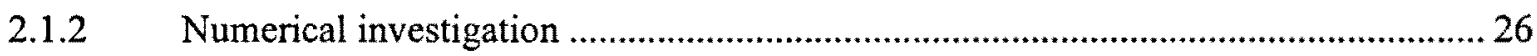

Chapter 3

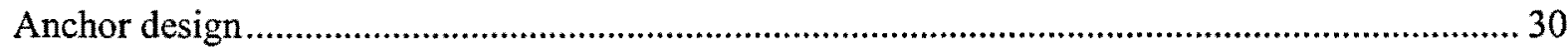

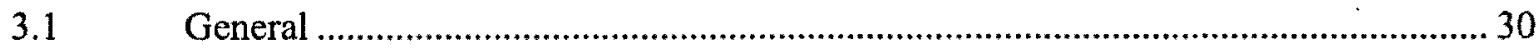

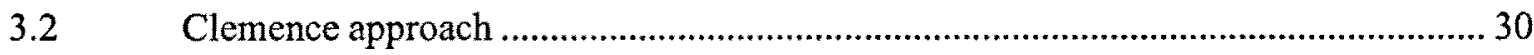

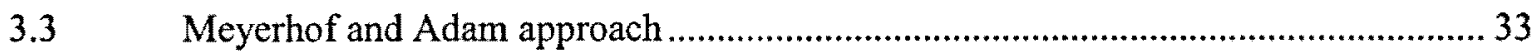

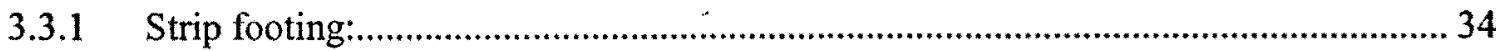

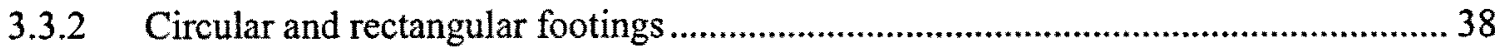

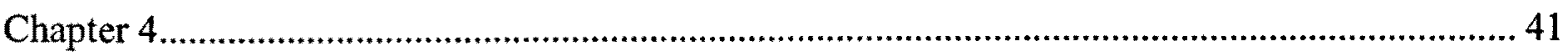

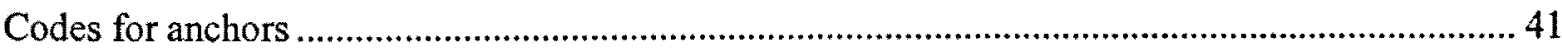

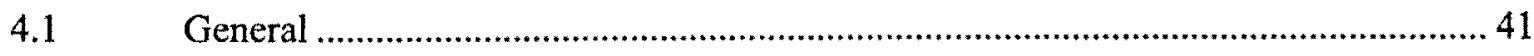

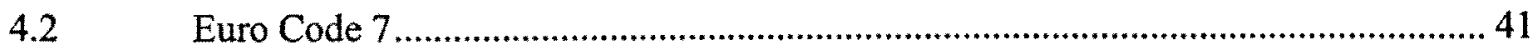




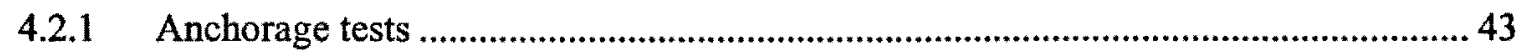

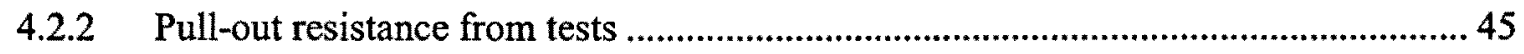

Chapter 5

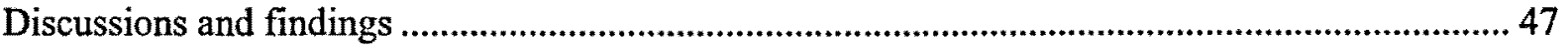

5.1 Comparison of Ilamparuthi's test results with Meyerhof's design equations ............. 47

$5.2 \quad$ Application of Euro code for plate anchors.................................................................. 48

5.3 Influence of sand density and H/D ratio on ultimate uplift capacity of anchors........ 49

Chapter 6

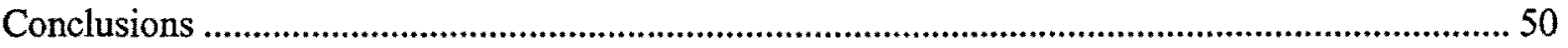

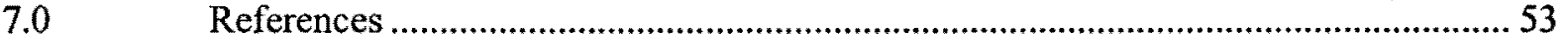




\section{List of Figures}

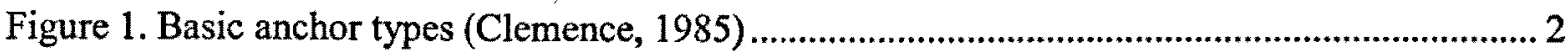

Figure 2. Three different failure modes in an earth anchor (Liu at. el., 2010) .................................. 3

Figure 3. Displacement fields in Lochaline (fine sand) and $\mathrm{L}$. Buzzard (coarse sand) $\mathrm{D} / \mathrm{B}=4$, $\mathrm{RD}=75 \%$ (Bouazza, 1993)

Figure 4. Displacement fields in Buzzard (sub-rounded) and Douglasmuir (sub-angular) sand $\mathrm{D} / \mathrm{B}=4, \mathrm{ID}=\mathbf{7 5 \%}$ (Bouazza, 1993)

Figure 5. Displacement fields in Douglasmuir (uniform) sand and Hyndford (well graded) sand $\mathrm{D} / \mathrm{B}=4, \mathrm{ID}=15 \%$ (Bouazza, 1993) 7

Figure 6. Displacement fields in Lochaline sand, sand $\mathrm{D} / \mathrm{B}=4, \mathrm{ID}=15 \%$ and $\mathrm{ID}=75 \%$ (Bouazza 1993)

Figure 7. Delineation of rupture surface in half cut model test on shallow anchor in dense sand (Ilamparuthi et. al., 2002)

Figure 8. Delineation of rupture surface in half cut model test on deep anchor in dense sand (Ilamparuthi et. al., 2002) 9

Figure 9. Relationship between pullout load and displacement for shallow circular anchor in sand (Ilamparuthi et. al., 2002) 10

Figure 10. Relationship between pullout load and displacement for deep circular anchor in sand (Ilamparuthi et. al., 2002)

Figure 11. Variation of peak pullout load with depth of embedment for circular plate anchors in dense sand (Ilamparuthi et. al., 2002)

Figure 12. Variation of displacement at peak pullout load with embedment ratio (Ilamparuthi et. al., 2002) 13

Figure 13. Variation of peak pullout load with anchor diameter in dense sand (Ilamparuthi et. al., 2002)

Figure 14. Influence of density on peak pullout load for various anchor diameters (Ilamparuthi et. al., 2002)

Figure 15. Relationship between breakout factor and embedment ratio for circular plate anchors in dense sand (Ilamparuthi et. al., 2002)

Figure 16. Variation of breakout factor with the angle of shearing resistance of sand for various embedment ratios (Ilamparuthi et. al., 2002) 
Figure 17. Determination of critical embedment ratio, $(\mathrm{H} / \mathrm{D})_{\mathrm{CR}}$, from breakout factor for circular plate anchors in dense sand (Ilamparuthi et. al., 2002)

Figure 18. Comparison of measured breakout factor, $\left(\mathrm{N}_{\mathrm{q}}\right)_{\mathrm{M}}$ and empirically estimated breakout factor, $\left(\mathrm{N}_{\mathrm{q}}\right) \mathrm{E}$, in medium-dense sand (Ilamparuthi et. al., 2002).

Figure 19. Comparison of breakout factors from empirical equations and previous experimental research for circular plate anchors in loose to medium-dense sand (Ilamparuthi et. al., 2002)

Figure 20. Comparison of breakout factors from empirical equations and previous experimental research for circular plate anchors in medium-dense to dense sand (Ilamparuthi et. al., 2002)

Figure 21. Displacement at peak pullout load for circular plate anchors at various embedment ratios in dense sand (llamparuthi et. al., 2002)

Figure 22. Determination of critical embedment ratio for circular plate anchors in dense sand (Ilamparuthi et. al., 2002),

Figure 23. Comparison of alternative estimations of critical embedment ratio for circular plate anchors in sand (llamparuthi et. al., 2002).

Figure 24.Uplift resistance-displacement curves in sand: (a) dense; (b) medium; (c) loose (Sakai \& Tanaka, 2007)

Figure 25.Comparison of laboratory and numerical results on pullout load-displacement relationship at different embedded depths. $Q_{r}$, shaft resistance; $Q_{q}$, end resistance (Hsu et. al., 1998).

Figure 26. Modified $\mathrm{N}_{\mathrm{q}}$ bearing capacity factor (Clemence, 1985) ................................................ 33

Figure 27. Failure of soil above a strip footing under uplift load (Meyerhof et. al., 1968) ............. 34

Figure 28. Theoratical uplift coefficients of earth pressure for strip footing (Meyerhof et. al., 1968)

Figure 29. Comparison of theory and model tests for footings in sand (Meyerhof et. al., 1968) ... 40 


\section{List of Tables}

Table 1.Comparison of breakout factors measured from field tests $\left(\mathrm{N}_{\mathrm{q}}\right)_{M}$ with those estimated from empirical equations $\left(\mathrm{N}_{\mathrm{qf}}\right)$ E (Ilamparuthi et. al., 2002).........................20

Table 2.Critical embedment ratios for circular plate anchors determined by various methods (Ilamparuthi et. al., 2002).......................................................25

Table 3.Comparison of critical embedment ratios obtained from the Ilamparuthi's investigation with those from published results (llamparuthi et. al., 2002) .26

Table 4.The value of $\mathrm{H} / \mathrm{B}$ form test results (Meyerhof et. al., 1968). .37

Table 5.Values of shape factor ' $s$ ' and coefficient $m$ against friction angle (Meyerhof et. al., 1968).

Table 6.Suggested values of $\zeta_{\mathrm{a}}$ (Andrew et. al., 2008). .46

Table 7.Comparison of Ilamparuthi's test results on shallow anchors in dense sand with Meyerhof's design formula 


\section{Chapter 1}

\section{Introduction}

\subsection{General}

Plate anchors, referred as anchor in this report, are foundation systems that are designed primarily to resist uplift (tensile) loads. Anchors are used to support structures such as transmission towers, anchored bulkheads, submerged pipelines, and tunnels. Research into the uplift resistance of an anchor provides algorithm for soil structure interaction problem. Various studies of anchors have been conducted by many researchers in order to understand anchor behavior but the discrepancies between model prediction and actual measurements are still varying extensively. It is believed that these discrepancies are due to the lack of full understanding of both anchor behavior and its interaction with surrounding soil during anchor uplifting (Liu at. el., 2010). Because of lack of theoretical or experimental verification for different anchor types the designers rely heavily on rules of thumb, manufacturers recommendations, or contractors experience in similar conditions.

In this report a number of model uplift tests and numerical investigations made by different authors are described and based on these readings the uplift behavior of anchors in sand is explored and anchor's design procedure is described. The factors which influence the design of anchors are explored. Basic anchor types, failure modes in anchors, and design codes are mentioned. To verify the design methods, published laboratory test data for circular anchors has been used in Meyerhof's proposed anchor design equations and results are compared with the laboratory results and are found to be comparable. This report focuses only on anchors embedded in sand. 


\subsection{Background}

\subsubsection{Types of anchors}

Anchors can be classified in three basic categories: Spread, helical, and grouted as shown in Figure

1. These basic anchor types differ primarily as a function of geometry and construction procedure.

Because of length limitations, in our case, the focus is only on spread anchors embedded in sand.

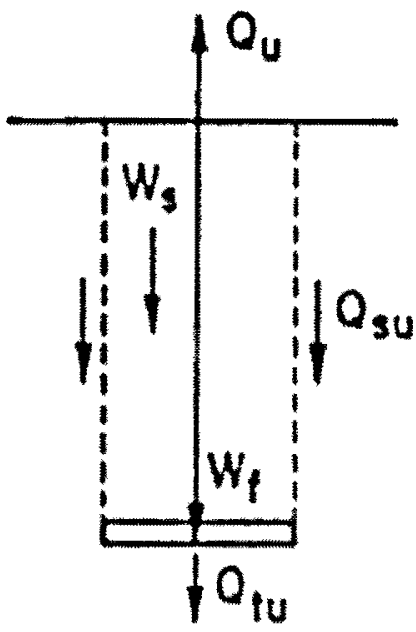

a) Spreod

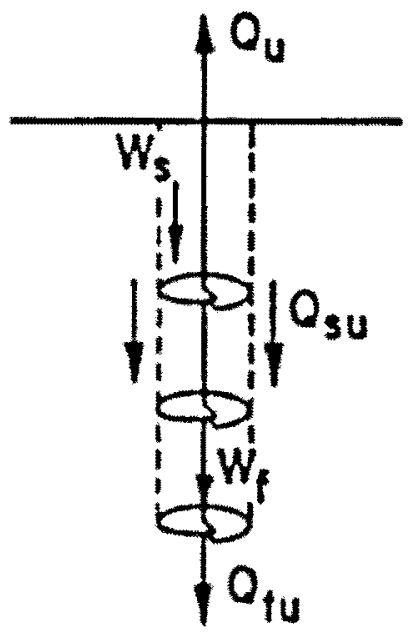

b) Helical

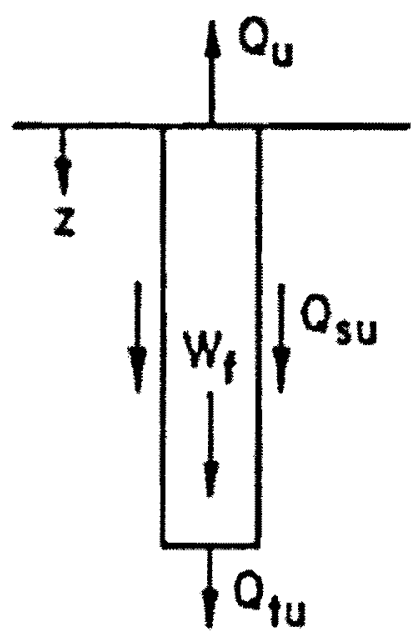

c) Grouted

Figure 1. Basic anchor types (Clemence, 1985)

\subsubsection{Anchors behavior pattern}

Although the previous section might suggest that there are fundamentally different patterns of behavior for the various anchor types, but the detailed examination of the available laboratory and field data indicates that the patterns are the same and variations occur in details. Consider the force-equilibrium diagram shown in Figure 1 . In each case the uplift capacity is given by:

$Q_{U}=W+Q_{t u}+Q_{s u}$

Where;

$\mathrm{Q}_{\mathrm{U}}=$ Uplift capacity 
$W=$ Weight of foundat;ion and enclosed soil $\left(W_{s}+W_{f}\right)$

$\mathrm{Q}_{\mathrm{tu}}=$ Tip resistance, and

$\mathrm{Q}_{\mathrm{su}}=$ Side resistance

\subsubsection{Failure modes in anchors}

Among various failure surfaces, there are mainly three distinctive failure modes proposed by several researchers, as shown in Figure2. The first failure surface is a frictional cylinder, as shown in Figure 2a, which was first proposed by Majer (1955). The pullout capacity is computed from the weight of soil within the cylindrical failure surface directly above the anchor plus the frictional resistance along this surface. Since the soil mobilized by an anchor is normally larger than the cylinder above the anchor, the pullout capacity tends to be underestimated based on this failure surface.

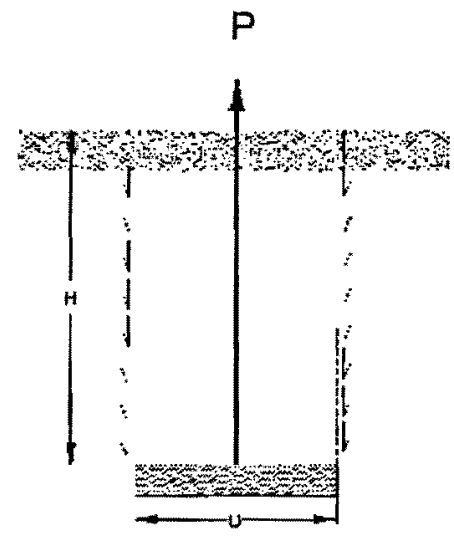

a)

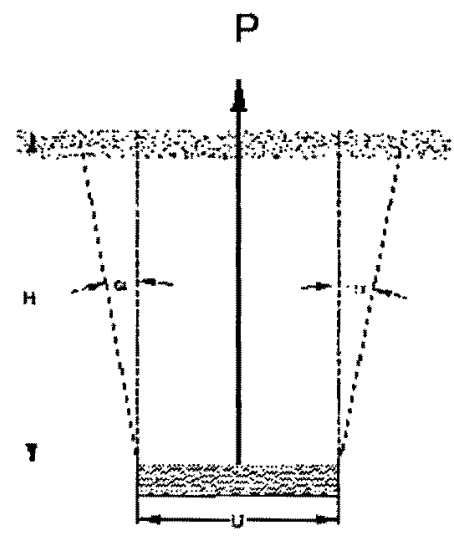

b)

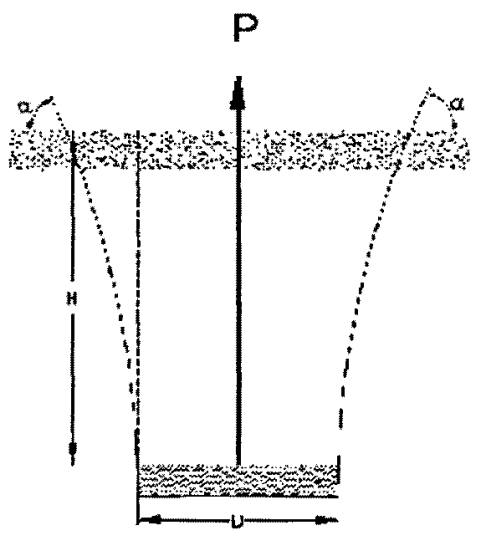

c)

Figure 2. Three different failure modes in an earth anchor (Liu at. el., 2010)

The second type of failure surface proposed firstly by Mors (1959), is a truncated cone that extends from the anchor with an apex angle of $90^{\circ}+\phi$, where $\phi$ is the friction angle of soil, as shown in Figure $2 b$. The pullout capacity is calculated to be only the weight of the soil within the 
truncated cone. The Mors' method is usually conservative for shallow anchors by ignoring the frictional force along the failure surface, but overestimates the pullout capacity in deep anchor cases, where the failure surface normally does not extend to the ground surface and will be smaller than the assumed truncated cone. The third type of failure surface is a circular surface extending from the edge of the anchor and intersecting the ground surface with an angle of approximately $45^{\circ}-\phi / 2$ as shown in Figure 2c. This type of failure surface was observed in many studies (Balla, 1961; Baker \& Kondner, 1966). According to this method the uplift capacity is given by the weight within the curved zone plus the shearing resistance along the curved surface. These assumptions are reasonable in case of shallow anchors but not applicable to deep anchors. This method over estimates the capacity in case of loose normally consolidated sand and under estimates in case of dense heavily consolidated sand (Liu at. el., 2010).

\subsection{Objectives}

The main objectives of the study are:

(i) To review existing research conditions related to anchors in sand.

(ii) To determine the uplift behaviour of anchors embedded in sand.

(iii) To determine the factors which influence the design of anchors embedded in sand.

(iv) To verify the proposed design methods by using published laboratory test results.

(v) To study available anchor codes. 


\section{Chapter 2}

\section{Literature review}

\subsection{Current research}

The focus of this research is to find out the factors which influence the uplift capacity of anchors embedded in sand. In order to find out the factors which influence the design of anchors it is important to study the experimental investigations or laboratory tests made on anchors embedded in sand. To achieve this, a number of model uplift tests, including laboratory tests and numerical investigations, made by different authors were studied which are described in the section 2.1.1.

\subsubsection{Laboratory testing and analysis}

\subsubsection{Effect of sand grain size, grain shape, sand grading, and density on uplift behavior} Bouazza (Bouazza, 1993) studied the effect of sand properties (grain size, grain shape, and grading) on the displacement fields and monitored their development around a shallow plate anchor subjected to an uplift load. He found that the displacement fields are influenced by the shape of the sand grains and sand grading. The magnitudes of the sand displacements were also found to be regulated to a large extent by the sand relative density (RD). Based on the test results it was concluded that the shape of displaced sand mass was independent of the grain size at a given depth, D, and relative density as shown in Figure 3, where ' $\mathrm{B}$ ' stands for diameter of the anchor plate. But the grain shape did influence the displacement field extending it laterally more in case of sub-angular shape grains than sub-rounded grains at a given relative density as shown in Figure 4. 

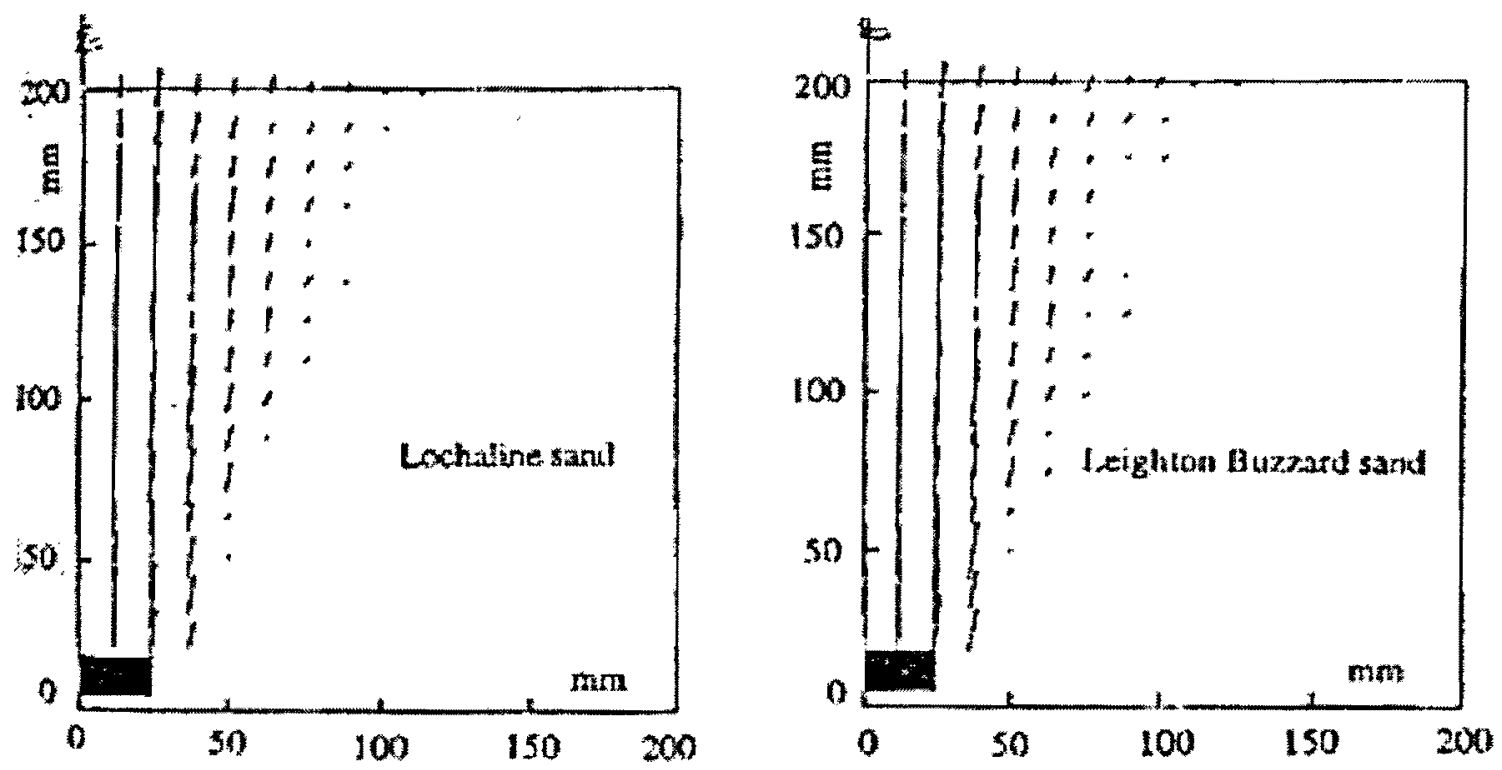

Figure 3. Displacement fields in Lochaline (fine sand) and $\mathrm{L}$. Buzzard (coarse sand) $\mathrm{D} / \mathrm{B}=4$, $\mathrm{RD}=75 \%$ (Bouazza, 1993)

It was also found that sand grading was also responsible for a distinct change in the extent of the zone of disturbance. An extended lateral disturbed zone was observed in case of well graded sand than uniform sand as shown in Figure 5.
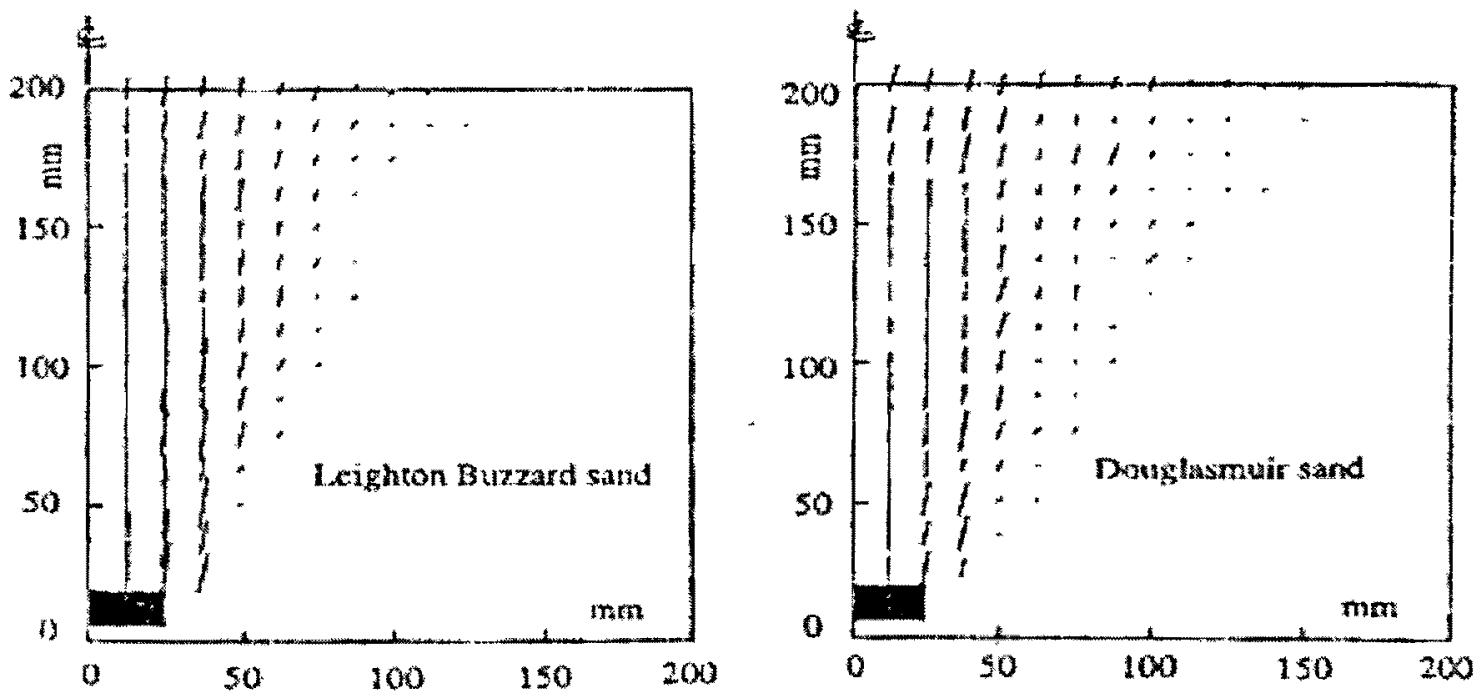

Figure 4. Displacement fields in Buzzard (sub-rounded) and Douglasmuir (sub-angular) sand $D / B=4, I D=75 \%$ (Bouazza, 1993) 

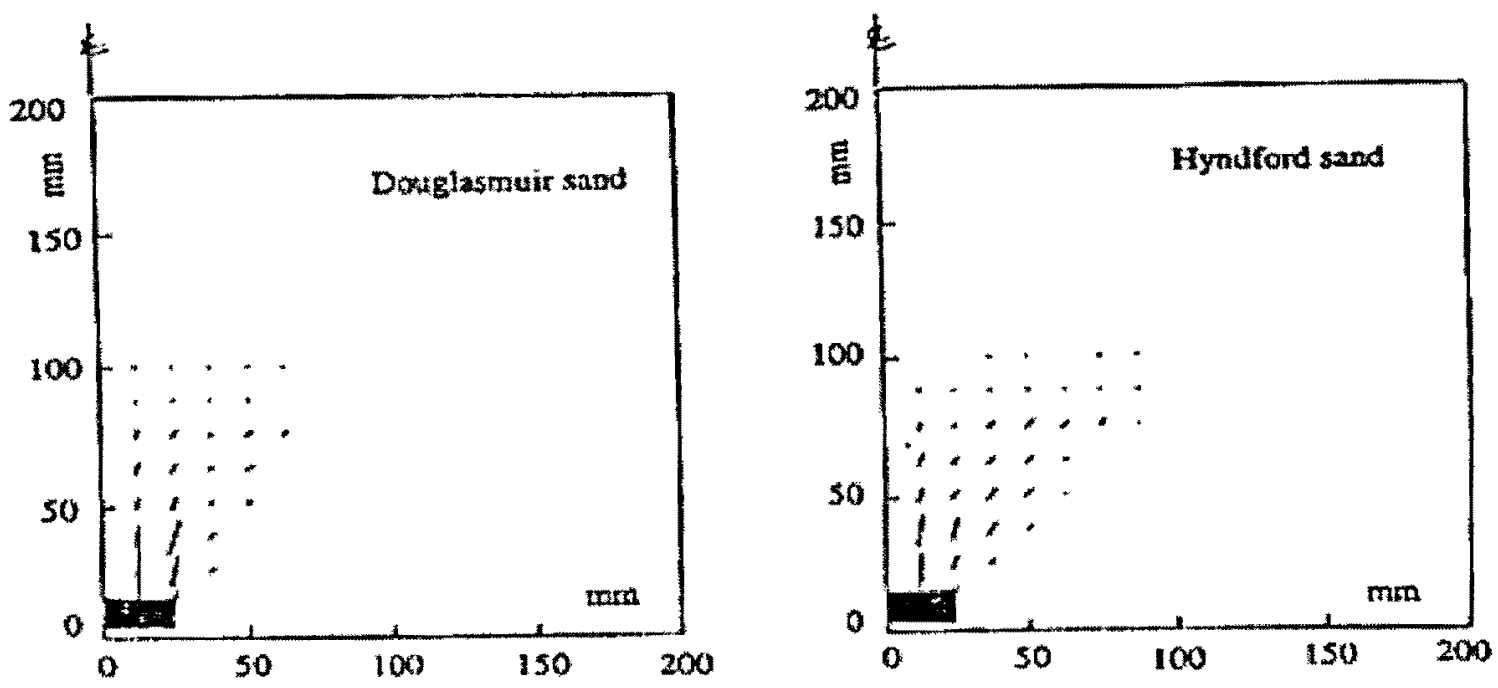

Figure 5. Displacement fields in Douglasmuir (uniform) sand and Hyndford (well graded) sand $D / B=4, I D=15 \%$ (Bouazza, 1993)
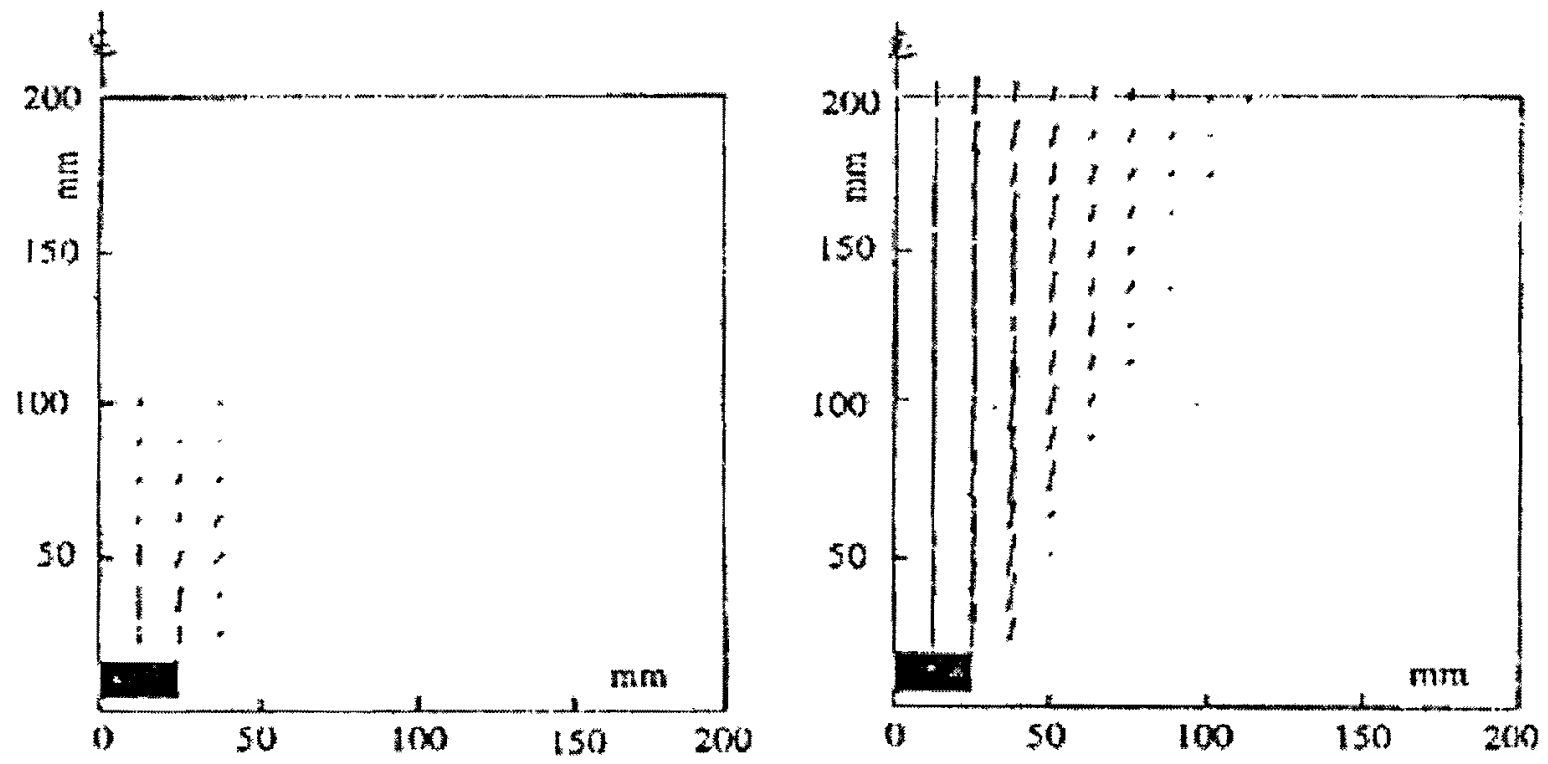

Figure 6. Displacement fields in Lochaline sand, sand $D / B=4, I D=15 \%$ and $I D=75 \%$ (Bouazza 1993)

The deformation of sand surrounding the anchor plate was also found to be regulated to a large extent by the sand relative density as shown in Figure 6 . In loose state the resulting deformation of 
the sand mainly occurred at 2.0B above the level of the plate and did not exceed $0.125 \mathrm{~B}$ to the side of the plate anchor. The magnitude of displacement gradually decreased from the plate to the sand surface and discontinuity became indistinct. The local shear failure was observed and the displacement field did not extend to the surface. The fact that the anchor movement disturbed only a small area of sand shows, that the plate anchor started to punch through the soil mass without encountering any resistance. It was noticed that $55 \%$ of the anchor displacement occurred during the application of the final $25 \%$ of the maximum load. In case of dense sand the zone of disturbed sand radiated from $0.25 \mathrm{~B}$ to $2.0 \mathrm{~B}$ to the side of the plate anchor indicating the deformation of the sand surrounding the plate anchor are regulated to large extent by the sand relative density.

\subsubsection{Delineation of rupture surface in dense sand for shallow anchors}

In tests on shallow half-cut models, with a H/D ratio of 2, a gently curved rupture surface emerged from the top of the anchor to the sand surface at approximately $\phi / 2$ to the vertical, which is in agreement with the previous findings as shown in Figure 7 (Ilamparuthi et. al., 2002).

\subsubsection{Delineation of rupture surface in dense sand for deep anchor}

For a deep anchor, a balloon-shaped rupture surface emerged at $0.8 \phi$ to the vertical immediately above the anchor up to a height of $1.5 \mathrm{D}$, above which it was balloon shaped extending about $2.9 \mathrm{D}$ and 1.6D in the vertical and radial directions, respectively as shown in Figure 8. The embedment ratio at which this change in behaviour takes place has been defined as the critical embedment ratio, $(\mathrm{H} / \mathrm{D})_{\mathrm{CR}}$. The Critical ratios of $4.9,5.8$, and 6.9 were estimated for the model anchors in the loose, medium-dense, and dense sand beds respectively. 


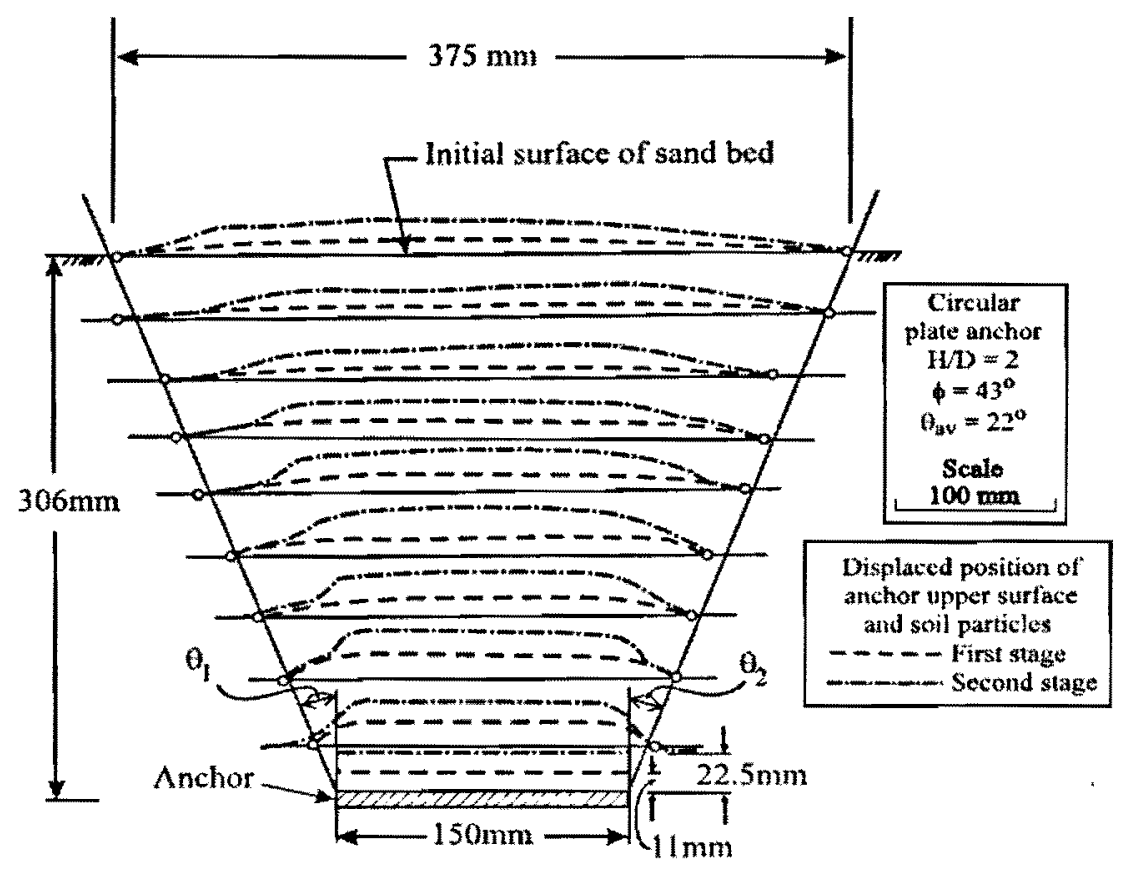

Figure 7. Delineation of rupture surface in half cut model test on shallow anchor in dense sand (Ilamparuthi et. al., 2002)

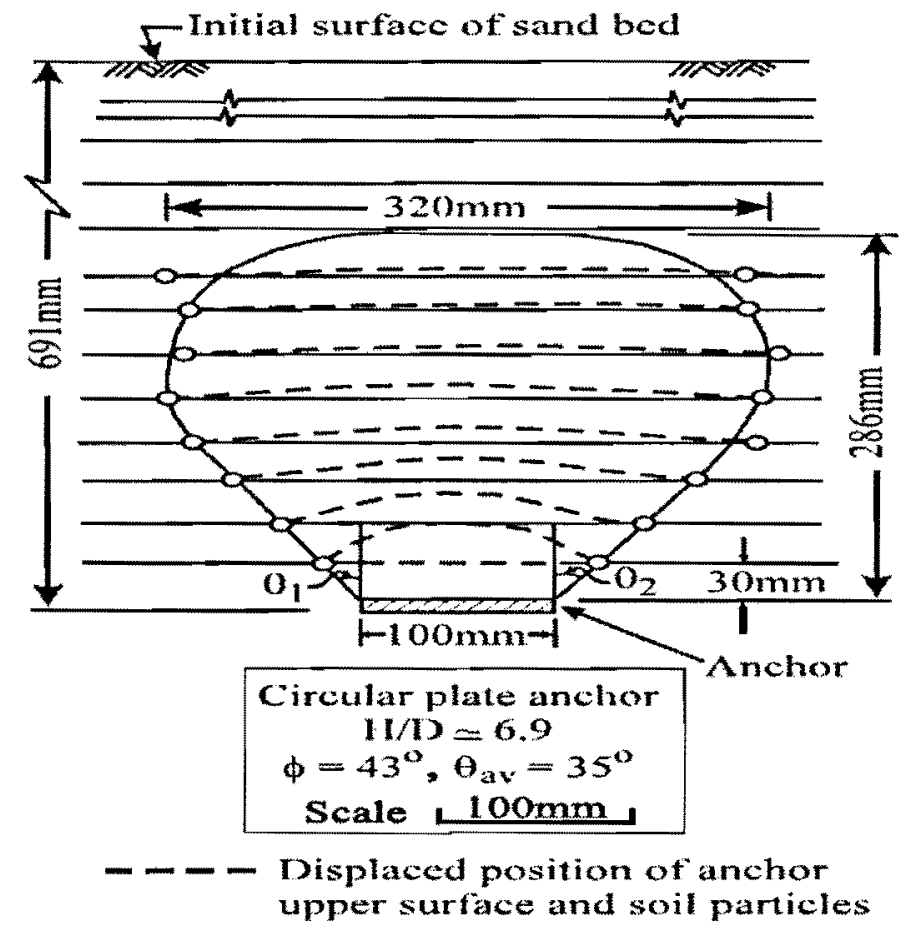

Figure 8. Delineation of rupture surface in half cut model test on deep anchor in dense sand (Ilamparuthi et. al., 2002) 


\subsubsection{Relationship between pullout load and displacement for shallow and deep circular anchor in sand}

The load-displacement behaviour for shallow circular anchor plates in sand was observed to be three phase exhibiting: 1) pre peak behaviour with a rapid increase in load, 2) post peak behaviour with a rapid load reduction, and 3) residual behaviour associated with a moderate decrease in load at large displacements as shown in Figure 9. Two-phase, load-displacement, behaviour was observed in deep circular anchor plates in sand, characterized by gradually increasing pre-peak trend followed by a very slowly decreasing residual post-peak behaviour as shown in Figure 10.

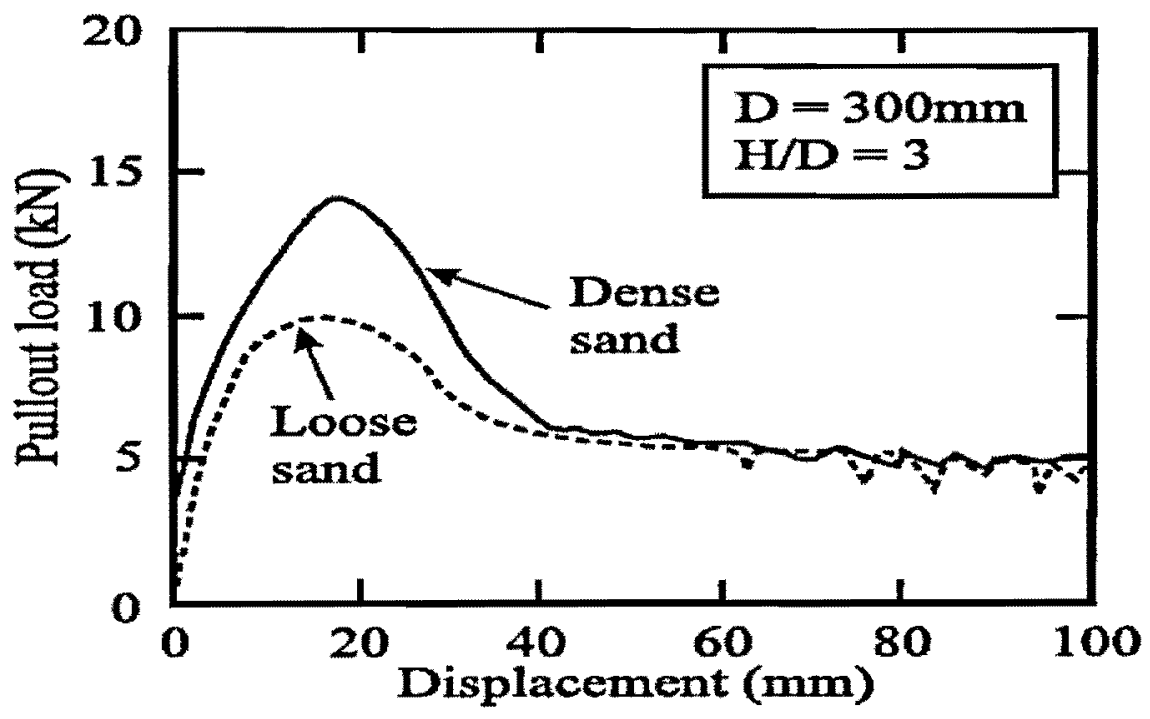

a) Shallow anchors

Figure 9. Relationship between pullout load and displacement for shallow circular anchor in sand (llamparuthi et. al., 2002)

2.1.1.5 Variation of peak pullout load with depth of embedment for circular plate anchors in dense sand

The variation of peak pullout load Qf with depth of embedment for all tests on anchors in dense sand is presented in Figure 11. The peak load increases at a higher rate with increased depth of embedment for a given anchor diameter. A similar, but less pronounced trend is found for anchors in loose and medium-dense sand. For example, the peak pullout loads for an anchor with $D=100$ 


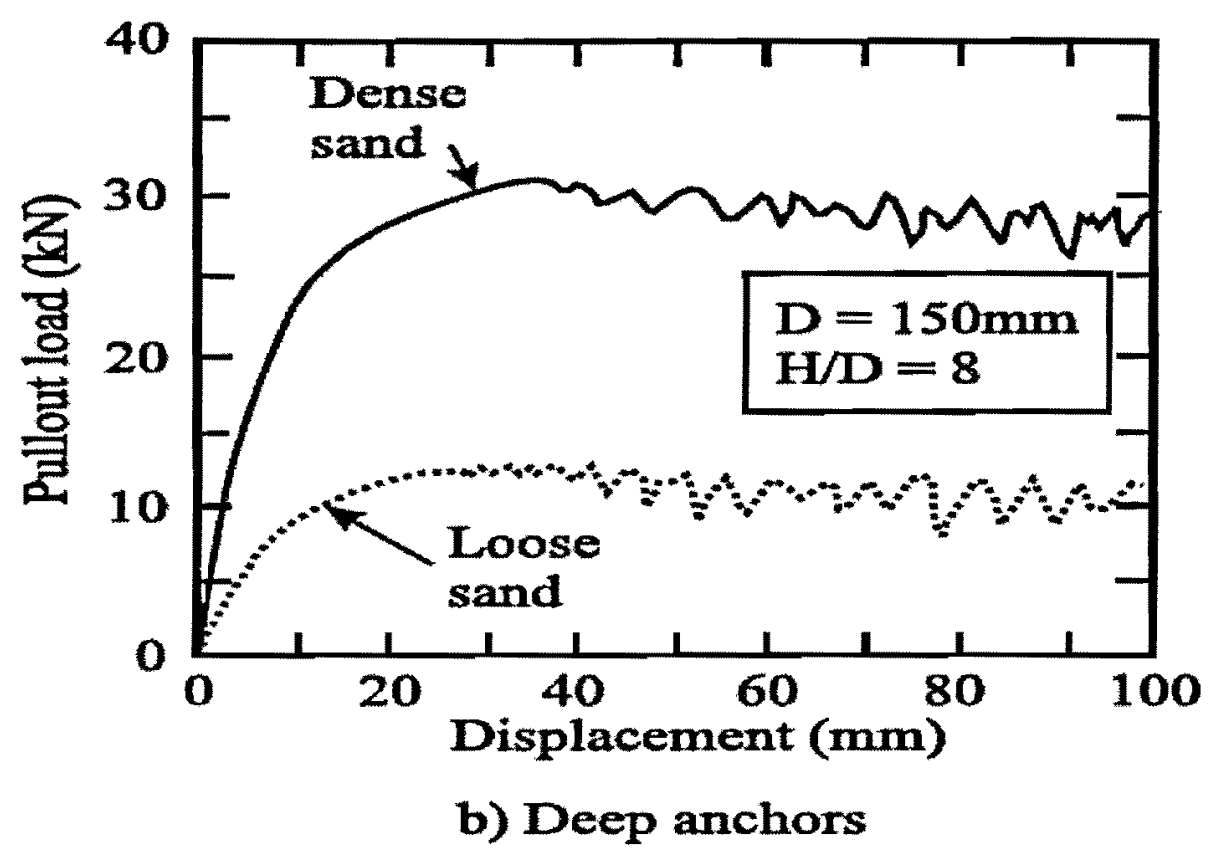

Figure 10. Relationship between pullout load and displacement for deep circular anchor in sand (Ilamparuthi et. al., 2002)

$\mathrm{mm}$ in loose, medium-dense, and dense sand are $2.0,2.6$, and $3.0 \mathrm{kN}$, respectively, at an embedment depth of $600 \mathrm{~mm}$ and $8.8,15.3$, and $23.2 \mathrm{kN}$, respectively, at an embedment depth of $1200 \mathrm{~mm}$. Thus the peak pullout load in dense sand is $50 \%$ greater than that in loose sand at the shallower depth $(H / D=6)$ and 2.6 times that at the greater depth $(H / D=12)$.

\subsubsection{Variation of displacement at peak pullout load with embedment ratio}

Displacements corresponding to peak loads in tests on $100 \mathrm{~mm}$ diameter anchors in loose and dense sand are shown in Figure 12. At shallow and intermediate depths the displacements are essentially the same, whereas at greater depths $(\mathrm{H} / \mathrm{D}>6)$ displacements in dense sand are significantly greater than those in loose sand. 


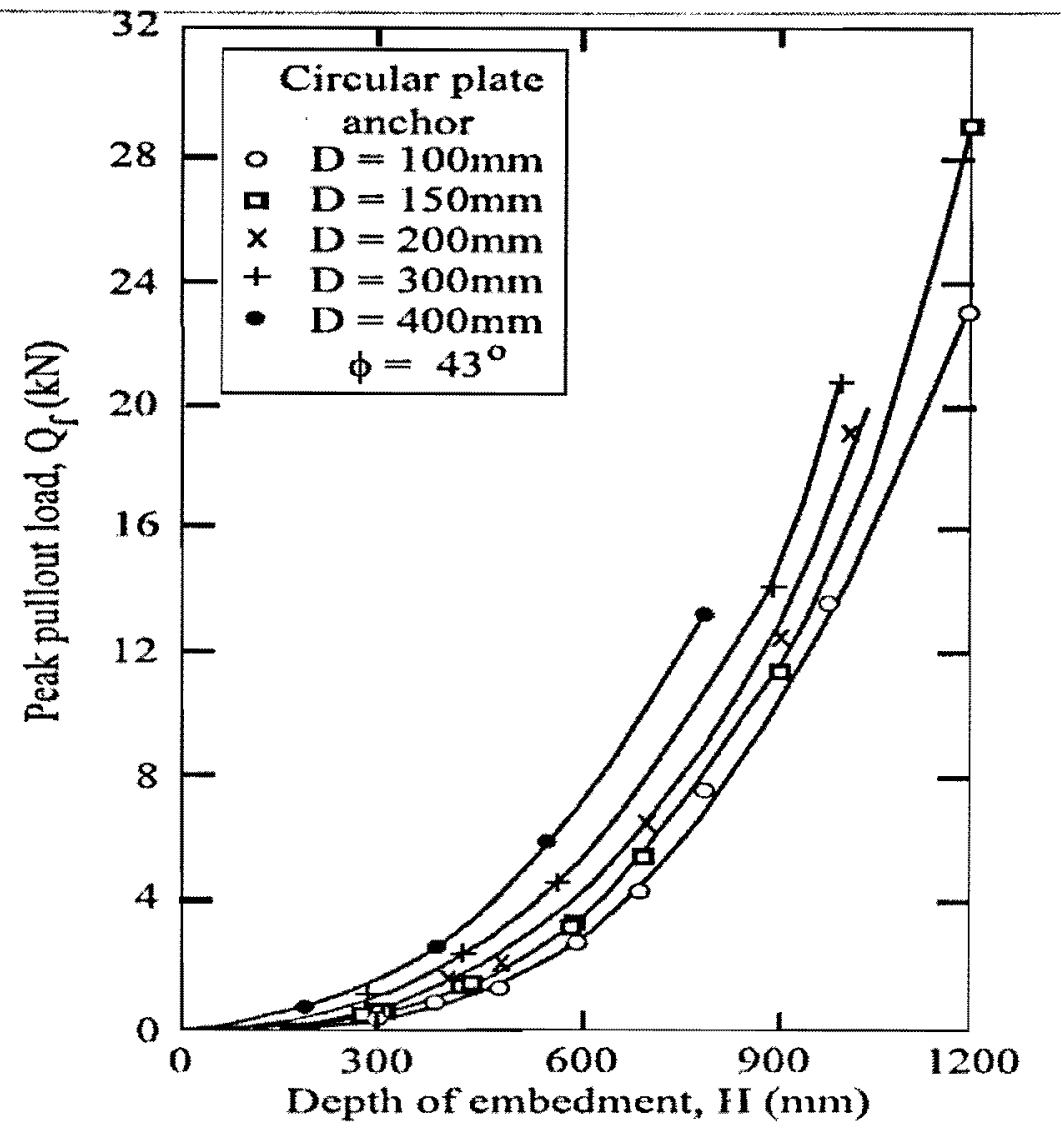

Figure 11. Variation of peak pullout load with depth of embedment for circular plate anchors in dense sand (llamparuthi et. al., 2002)

\subsubsection{Variation of peak pullout load with anchor diameter in dense sand}

Peak pullout loads for tests in dense sand shown in Figure 13 increase linearly with an increase in

D for each depth of embedment $\mathrm{H}$ for the range of diameters studied. The rate increases with an increase in $\mathrm{H}$, but Qf is not a simple multiple of $\mathrm{D}$. 


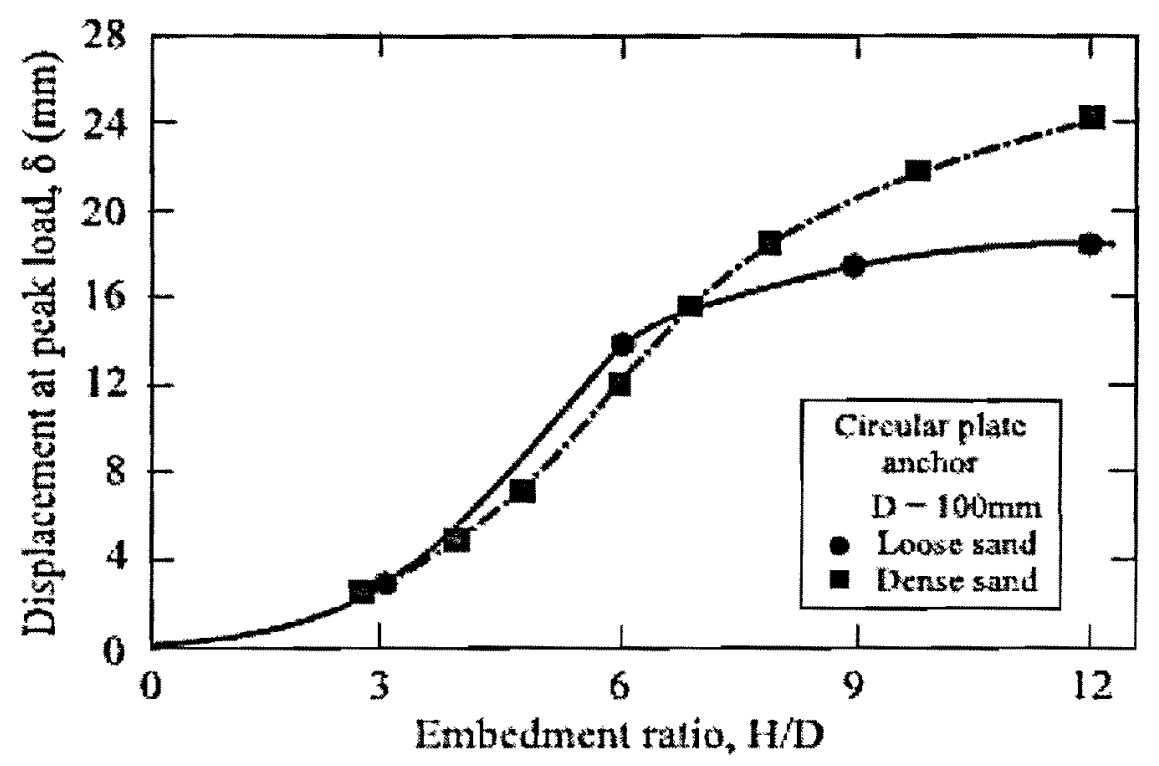

Figure 12. Variation of displacement at peak pullout load with embedment ratio (Ilamparuthi et. al., 2002)

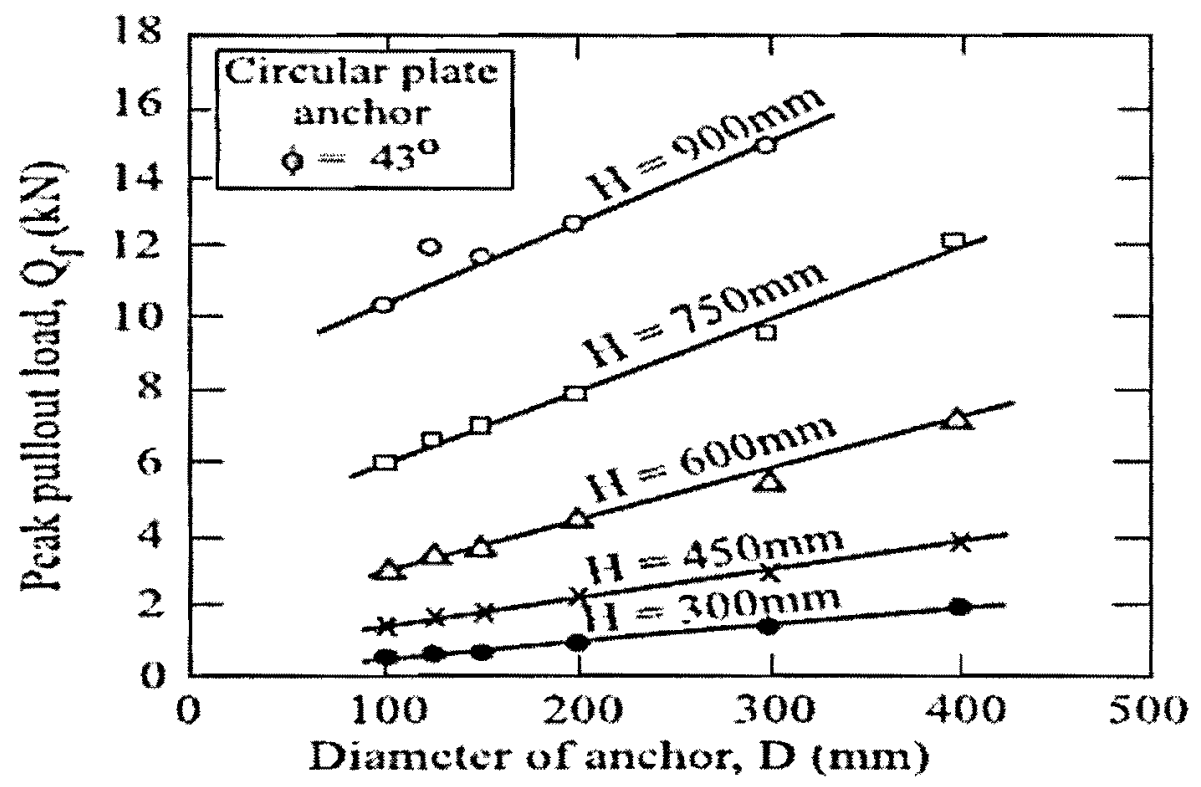

Figure 13. Variation of peak pullout load with anchor diameter in dense sand (llamparuthi et. al., 2002) 


\subsubsection{Influence of density on peak pullout load for various anchor diameters}

A typical variation of interpolated peak pullout loads for various diameters at a depth of $750 \mathrm{~mm}$ is presented in Figure 14 to illustrate the influence of density. For a given H and D as friction angle increases Qf increases.

\subsubsection{Relationship between breakout factor and embedment ratio for circular plate anchors} in dense sand

The peak pullout load, $\mathrm{Q}_{\mathrm{f}}$, is commonly expressed as a dimensionless breakout factor, $\mathrm{N}_{\mathrm{gf}}$, defined as follows.

$\mathrm{N}_{\mathrm{qf}}=\mathrm{Q}_{\mathrm{f}} / \gamma^{\prime} \mathrm{AH}$

where $A$ is the plan area of the anchor and $y^{\prime}$ is the effective unit weight of the sand bed. Breakout factors for anchors in dense sand are presented in Figure 15.

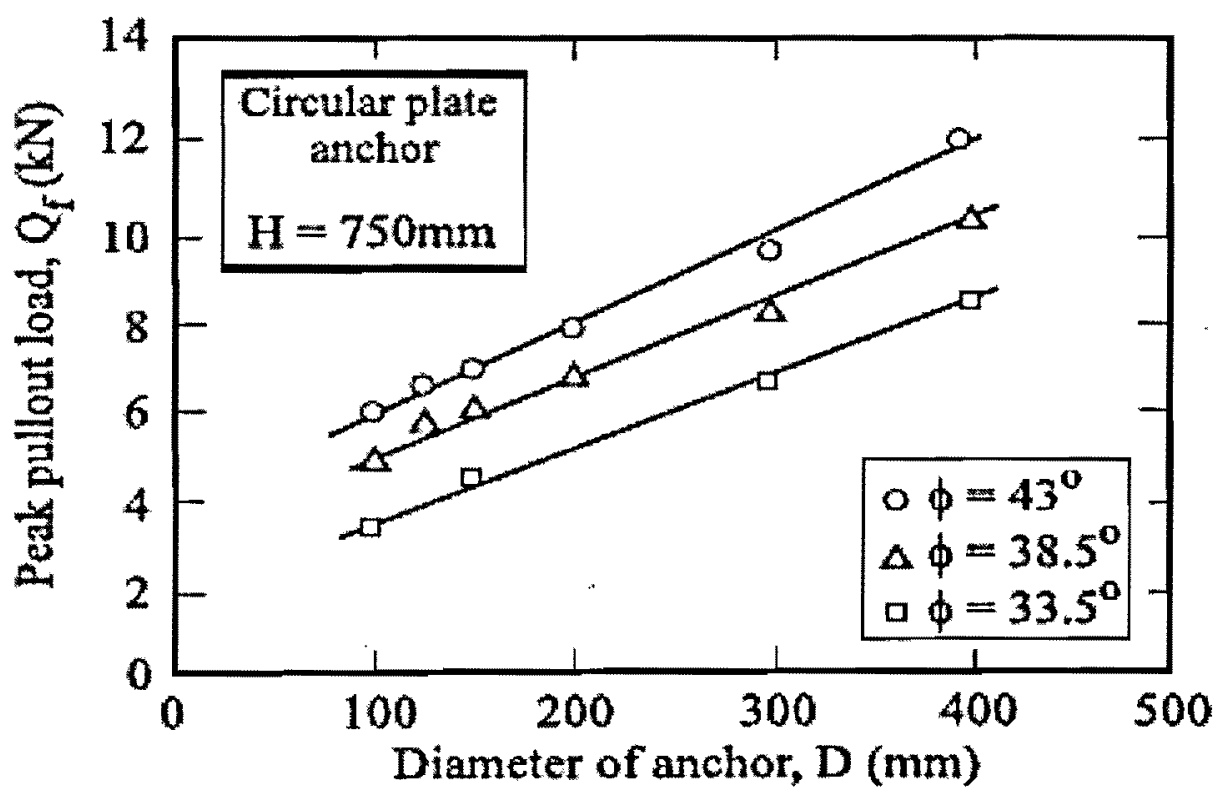

Figure 14. Influence of density on peak pullout load for various anchor diameters (llamparuthi et. al., 2002) 


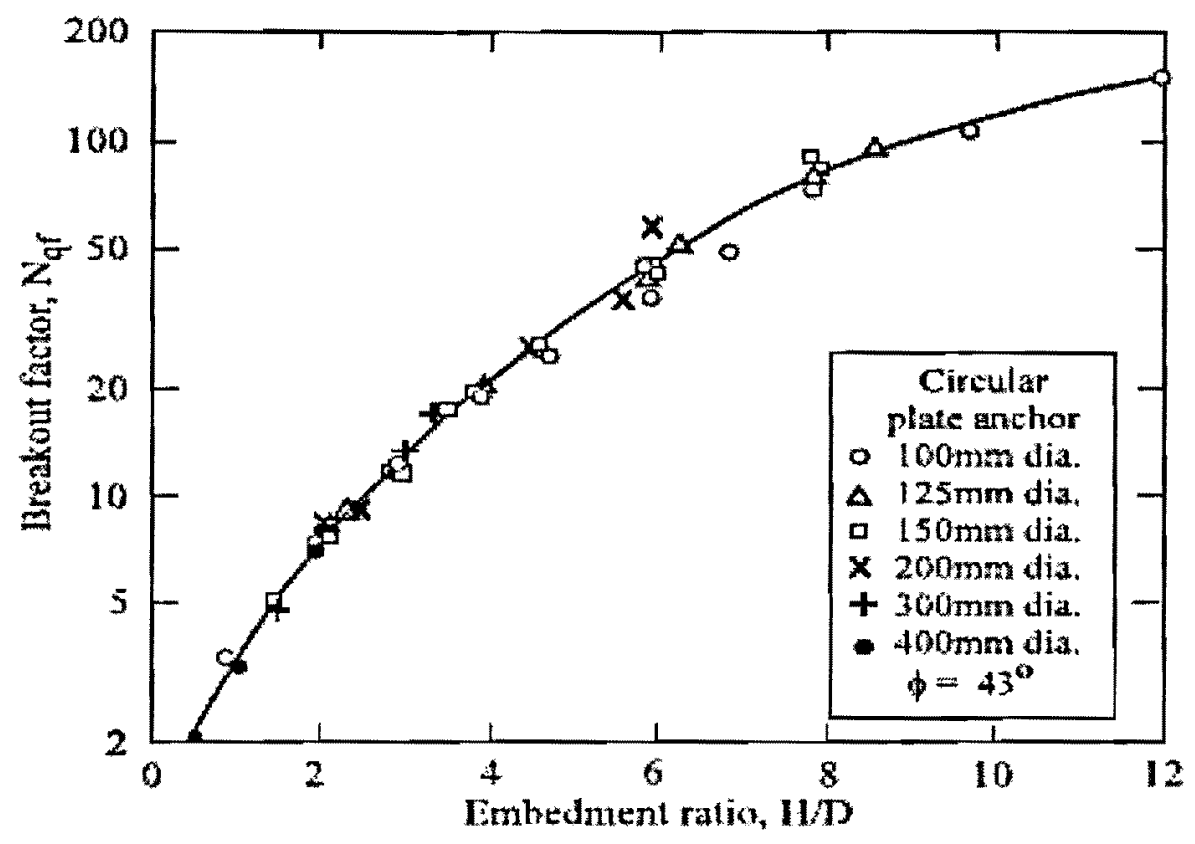

Figure 15. Relationship between breakout factor and embedment ratio for circular plate anchors in dense sand (Ilamparuthi et. al., 2002)

The results shown in Figure 15 indicates that, for a given density of soil, $\mathrm{N}_{\mathrm{qf}}$ is dependent only on $\mathrm{H} / \mathrm{D}$ and is relatively unaffected by $\mathrm{D}$ for the range of anchor diameters investigated. Similar relationships are obtained for anchors in medium-dense and loose sand. It should be noted, however, that in comparing tests on much smaller $25-50 \mathrm{~mm}$ models a reduction in $\mathrm{N}_{\mathrm{qf}}$ does occur with an increase in D. The breakout factor tends to become asymptotic to the embedment ratio (H/D) axis beyond $H / D=11$ and $H / D=10$ for medium-dense and loose sands, respectively, but is still increasing at $\mathrm{H} / \mathrm{D}=12$ for dense sand.

\subsubsection{Variation of breakout factor with the angle of shearing resistance of sand for various embedment ratios}

Figure 16 shows the variation of $\mathrm{N}_{\mathrm{qf}}$ with $\phi$ for various $H / D$ values. The relationships are linear but exhibit higher rates of increase of $\mathrm{N}_{\mathrm{qf}}$ with $\phi$ at higher $\mathrm{H} / \mathrm{D}$ value. The breakout factor for any $\phi$ and $\mathrm{H} / \mathrm{D}$ for the range studied (up to $\mathrm{H} / \mathrm{D}=12$ ) may therefore be determined. The breakout factor is greater at higher soil densities for a given anchor embedment ratio. For example, an increase in 
$\phi$ value from 33.5 to $43^{\circ}$ increases the breakout factor by 40 and $100 \%$ at embedment ratios of 4.2 and 8.4 , respectively.

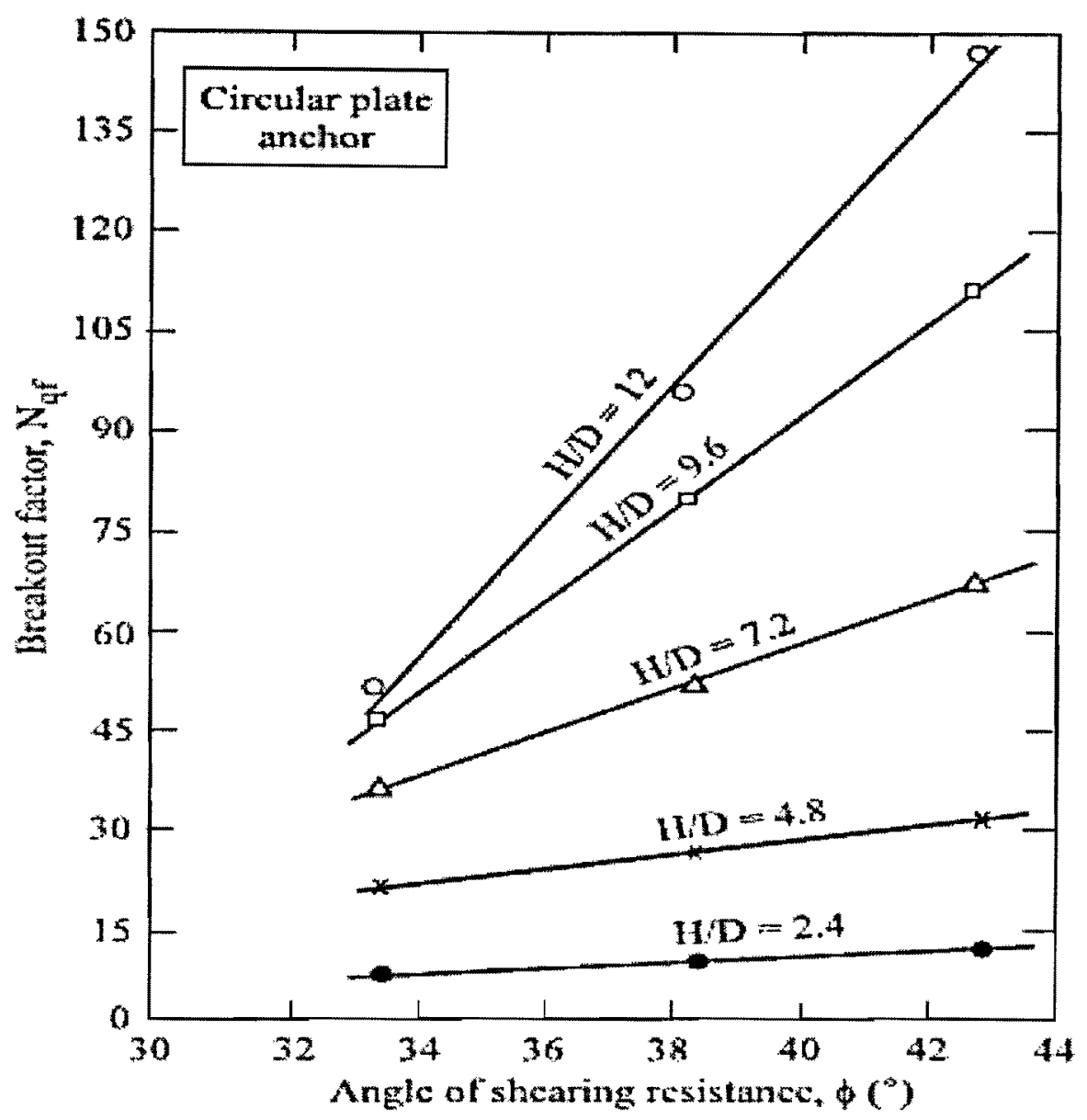

Figure 16. Variation of breakout factor with the angle of shearing resistance of sand for various embedment ratios (Ilamparuthi et. al., 2002)

2.1.1.11 Determination of critical embedment ratio, $(\mathrm{H} / \mathrm{D}) \mathrm{CR}$, from breakout factor for circular plate anchors in dense sand

The critical depth can be obtained from a logarithm-logarithm plot between $\mathrm{N}_{\mathrm{qf}}$ and $\mathrm{H} / \mathrm{D}$ as shown in Figure 17 for dense sand. There are changes in slope at embedment ratios of 1.6 and 6.6. The significance of the change of slope at $H / D=1.6$ is not yet understood. However, the change of slope at $\mathrm{H} / \mathrm{D}=6.6$ is broadly consistent with the critical embedment ratio observed in half-cut 
model tests $(\mathrm{H} / \mathrm{D}=6.9)$ and the change in the load-displacement behavior (see Figure 6) at about $\mathrm{H} / \mathrm{D}=6.3$. The critical embedment ratios were also determined in plots similar to Figure 16 for medium-dense and loose sand beds, and respective values of 5.5 and 4.5 were obtained. Critical embedment ratios of 5.9 and 4.0 were estimated for medium-dense and loose sands, respectively, from the shape of the load-displacement relationship. By comparison, the $\mathrm{H} / \mathrm{D}$ value at which the $\mathrm{N}_{\mathrm{qf}}$ tends to become constant, a criterion previously used to define critical depth, is about twice these embedment ratios. Alternative methods of determining $(\mathrm{H} / \mathrm{D})_{\mathrm{CR}}$ based on the displacement corresponding to peak pullout load and surface heave are examined later.

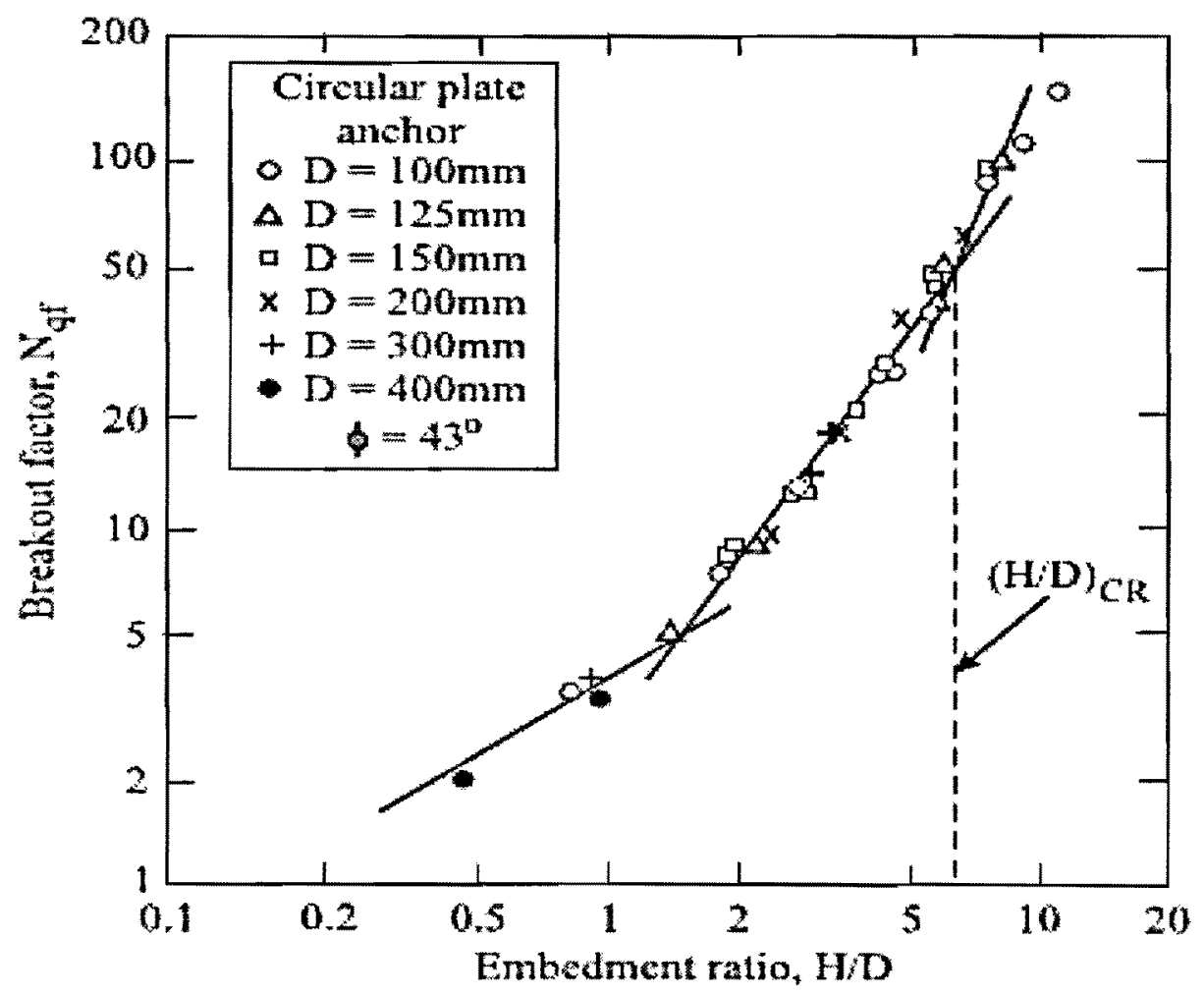

Figure 17. Determination of critical embedment ratio, (H/D) $\mathrm{CR}$, from breakout factor for circular plate anchors in dense sand (Ilamparuthi et. al., 2002) 


\subsubsection{Ilamparuthi,s proposed empirical design equations}

Ilamparuthi (Ilamparuthi et al. 2002), based on his research, formulated a set of equations for anchors in loose sand. The following empirical equations predict the breakout factor for any embedment ratio in loose sand in terms of $\mathrm{N}_{\mathrm{gf}}$ for a circular plate anchor with $\mathrm{H} / \mathrm{D}=1$.

$$
\begin{array}{ll}
N_{\mathrm{qf}}=\mathrm{e}^{(33.5 / 28)(\mathrm{H} / \mathrm{D})} & \text { for } 0 \leq(\mathrm{H} / \mathrm{D}) \leq 1.0 \\
\mathrm{~N}_{\mathrm{qf}}=(\mathrm{H} / \mathrm{D}) \mathrm{N}_{\mathrm{qf}} & \text { for } 1.0 \leq(\mathrm{H} / \mathrm{D}) \leq 2.4 \\
\mathrm{~N}_{\mathrm{qf}}=(\mathrm{H} / 2 \mathrm{D})\left(\mathrm{e}^{\tan \phi \ln (\mathrm{H} / \mathrm{D})}\right) \mathrm{N}_{\mathrm{qfl}} & \text { for } 2.4 \leq(\mathrm{H} / \mathrm{D}) \leq 4.2 \\
\mathrm{~N}_{\mathrm{qf}}=\left[(\mathrm{H} / \mathrm{D})+(\mathrm{H} / \mathrm{D})\left(\mathrm{e}^{\tan \phi \ln (\mathrm{D} / \mathrm{H})}\right)\right] \mathrm{N}_{\mathrm{qf} f} & \text { for } 4.2 \leq(\mathrm{H} / \mathrm{D}) \leq 6.0 \\
\mathrm{~N}_{\mathrm{qf}}=\left[(\mathrm{H} / \mathrm{D})+\left(\mathrm{e}^{\tan \phi \ln (\mathrm{H} / \mathrm{D})}\right)\right] \mathrm{N}_{\mathrm{qf}} & \text { for } 6.0 \leq(\mathrm{H} / \mathrm{D}) \leq 10.0 \\
\mathrm{~N}_{\mathrm{qf}}=\left[\mathrm{N}_{\mathrm{qf} 10}+\mathrm{e}^{\tan \phi \ln (\mathrm{HD}-10)}\right] & \text { for } 10.0 \leq(\mathrm{H} / \mathrm{D}) \leq 12.0
\end{array}
$$

where $\mathrm{N}_{\mathrm{qf}}$ is the breakout factor for any desired $\mathrm{H} / \mathrm{D}$ ratio in loose sand, $\mathrm{N}_{\mathrm{qfl}}$ is the breakout factor for $H / D=1.0$ (which is equal to 3.3) for $\phi=33.5^{\circ}$, and $N_{q 110}$ is the breakout factor for $H / D=10.0$. Breakout factors for anchors with $\mathrm{H} / \mathrm{D}<(\mathrm{H} / \mathrm{D})_{\mathrm{CR}}$ are obtained from Eqs. (2)-(4), and Eqs. (5)-(7) relate to deep anchors. The following equation can be used to predict the breakout factor for any embedment ratio and angle of shearing resistance for anchors in denser sands.

$\mathrm{N}_{\mathrm{qf}}{ }^{\phi}=\mathrm{N}_{\mathrm{qf}}^{33.5}\left(\mathrm{e}^{(\mathrm{H} / 3 \mathrm{D})(\phi-33.5) / 33.5}\right)$

where $\mathrm{N}_{\mathrm{qf}}$ is the breakout factor for any $\phi$ value and $\mathrm{H} / \mathrm{D}$ value, and $\mathrm{N}_{\mathrm{qf}}{ }^{33.5}$ is the breakout factor for $\phi=33.5^{\circ}$ at the same embedment ratio, obtained from Eqs. (2)-(7). 


\subsubsection{Comparison of measured breakout factor, $\left(\mathrm{N}_{\mathrm{q}}\right)_{M}$ and empirically estimated}

breakout factor, $\left(\mathrm{N}_{\mathrm{q}}\right)_{\mathrm{E}}$, in medium-dense sand

Breakout factors determined from the empirical equations (2)-(8) compare very well with the experimental values with average differences being $-1.0,-2.4$. and $+5.2 \%$ for loose, mediumdense, and dense sands, respectively. The comparison for anchors in medium-dense sand is shown in Figure 18.

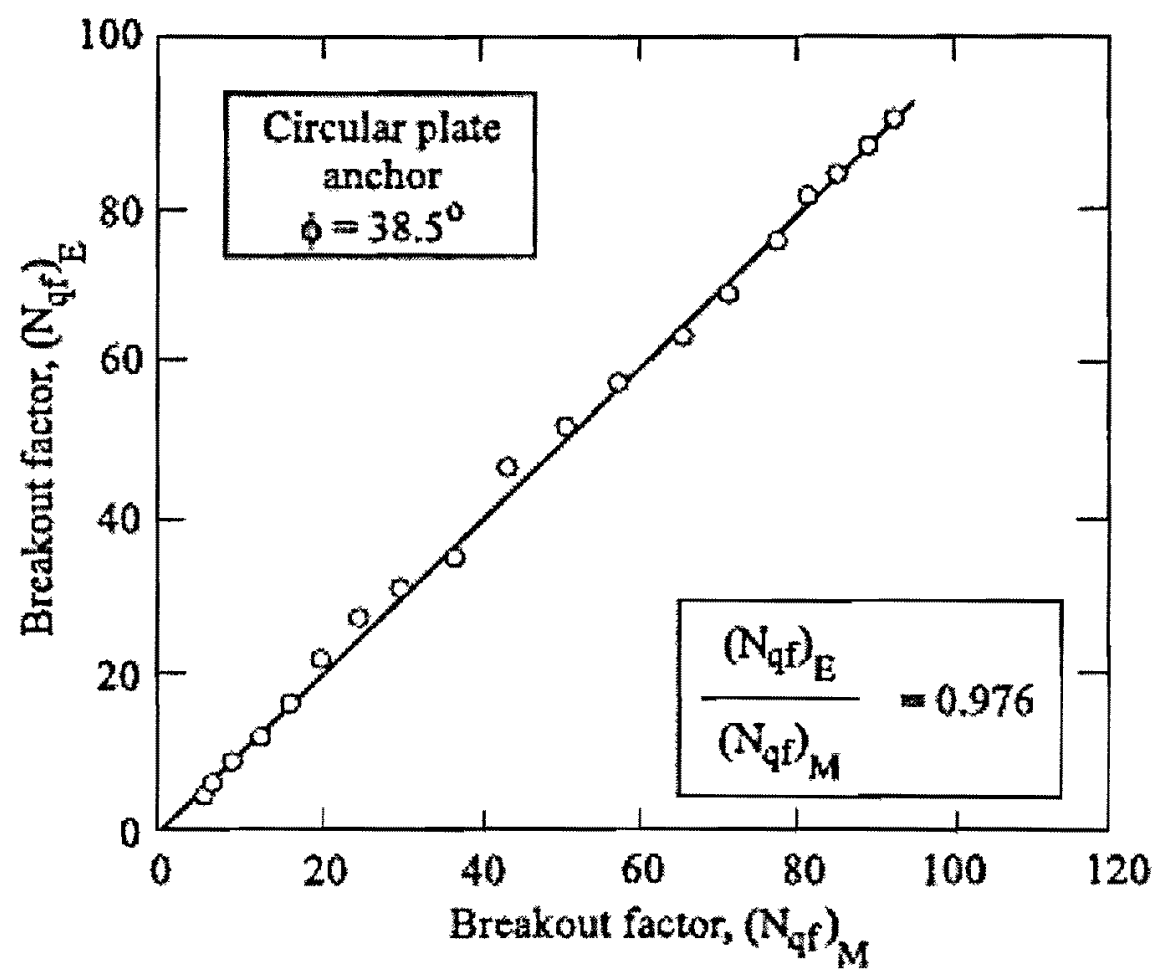

Figure 18. Comparison of measured breakout factor, $\left(\mathrm{N}_{\mathrm{q}}\right)_{\mathrm{M}}$ and empirically estimated breakout factor, $\left(\mathrm{N}_{\mathrm{q}}\right)_{\mathrm{E}}$, in medium-dense sand (llamparuthi et. al., 2002)

Table 1 shows the comparison between the breakout factors from the empirical equations and those obtained in the field for loose sand. The empirical breakout factors are comparable with values computed from published data. 
Table 1. Comparison of breakout factors measured from field tests $\left(\mathrm{N}_{\mathrm{q}}\right)_{\mathrm{M}}$ with those estimated from empirical equations $\left(\mathrm{N}_{\mathrm{q} f}\right)_{\mathrm{E}}$ (llamparuthi et. al., 2002).

\begin{tabular}{|c|c|c|c|c|c|c|c|}
\hline Aothors & $\begin{array}{c}\text { Diameter } \\
\mathrm{D}(\mathrm{m})\end{array}$ & $\begin{array}{c}\text { Depth, } \\
\mathrm{H}(\mathrm{m})\end{array}$ & $\mathrm{H} / \mathrm{D}$ & $\phi\left(^{\circ}\right)$ & $\left(\mathrm{N}_{\mathrm{qf}}\right) \mathrm{M}$ & $\left(\mathrm{N}_{\mathrm{qf}}\right) \mathrm{E}$ & $\left(\mathrm{N}_{\mathrm{q}}\right)_{\mathrm{M}}\left(\mathrm{N}_{\mathrm{q}}\right) \mathrm{E}$ \\
\hline \multirow{4}{*}{ Balla 1961 } & 1.900 & 1.50 & 0.79 & 36 & 3.2 & 2.62 & 1.22 \\
\cline { 2 - 8 } & 1.900 & 1.45 & 0.76 & 36 & 3.3 & 2.53 & 1.30 \\
\cline { 2 - 8 } & 1.300 & 2.50 & 1.92 & 30 & 3.8 & 5.92 & 0.64 \\
\cline { 2 - 8 } & 1.100 & 2.70 & 2.45 & 30 & 6.00 & 6.72 & 0.89 \\
\cline { 2 - 8 } & 1.400 & 2.50 & 1.78 & 30 & 3.90 & 5.52 & 0.71 \\
\hline \multirow{5}{*}{ Sutherland 1965 } & 2.390 & 2.44 & 1.02 & 45 & 3.52 & 3.71 & 0.95 \\
\cline { 2 - 8 } & 2.390 & 4.57 & 1.91 & 45 & 7.69 & 7.84 & 0.98 \\
\cline { 2 - 8 } & 2.390 & 4.57 & 1.91 & 45 & 7.37 & 7.84 & 0.94 \\
\cline { 2 - 8 } & 2.390 & 5.18 & 2.17 & 45 & 9.44 & 9.16 & 1.03 \\
\cline { 2 - 8 } & 2.390 & 5.18 & 2.17 & 45 & 9.22 & 9.16 & 1.00 \\
\cline { 2 - 8 } & 2.390 & 7.00 & 2.93 & 45 & 12.28 & 13.75 & 0.89 \\
\hline Baker and Konder & 0.305 & 1.63 & 5.33 & 37 & 33.4 & 28.20 & 1.18 \\
\hline \multirow{5}{*}{ Kananyan 1966 } & 0.406 & 3.48 & 8.56 & 37 & 48.00 & 56.40 & 0.85 \\
\cline { 2 - 8 } & 0.600 & 1.00 & 1.67 & 32 & 5.4 & 5.38 & 1.00 \\
\cline { 2 - 8 } & 0.800 & 1.00 & 1.25 & 32 & 4.18 & 4.07 & 1.02 \\
\cline { 2 - 8 } & 1.000 & 1.00 & 1.00 & 32 & 3.23 & 3.25 & 0.99 \\
\cline { 2 - 8 } & 1.200 & 1.00 & 0.83 & 32 & 2.73 & 2.67 & 1.02 \\
\hline Vesic 1971 & 0.406 & - & 8.20 & 37 & 40.00 & 53.60 & 0.75 \\
\hline \multirow{3}{*}{ Kawasniewski et al. } & 0.900 & 2.00 & 2.22 & 30 & 6.16 & 6.78 & 0.91 \\
\cline { 2 - 8 } & 0.700 & 1.90 & 2.71 & 28 & 6.93 & 7.45 & 0.93 \\
\cline { 2 - 7 } & 0.700 & 1.30 & 1.85 & 32 & 5.74 & 5.94 & 0.97 \\
\cline { 2 - 7 } & 0.700 & 1.50 & 2.14 & 32 & 7.02 & 6.84 & 1.03 \\
\hline
\end{tabular}

Breakout factors from Eqs. (2)-(8) are also compared graphically with laboratory model tests (Kwasniewski et al. 1975; Sutherland et al. 1982; Andreadis and Harvey 1981; Balla 1961; Baker and Kondner 1966; Clemence and Veesaert 1977; Murray and Geddes 1987; Bemben and Kupferman 1975) involving various anchor diameters, embedment depths, and sand conditions. The results are shown in Figure 19 for sand with $\phi$ values between 28 and $36^{\circ}$ (deemed to be loose to medium-dense sand) and in Figure 19 for $\phi$ values between 38 and $46^{\circ}$ (deemed to be mediumdense to dense sand). The results from empirical equations are comparable. 


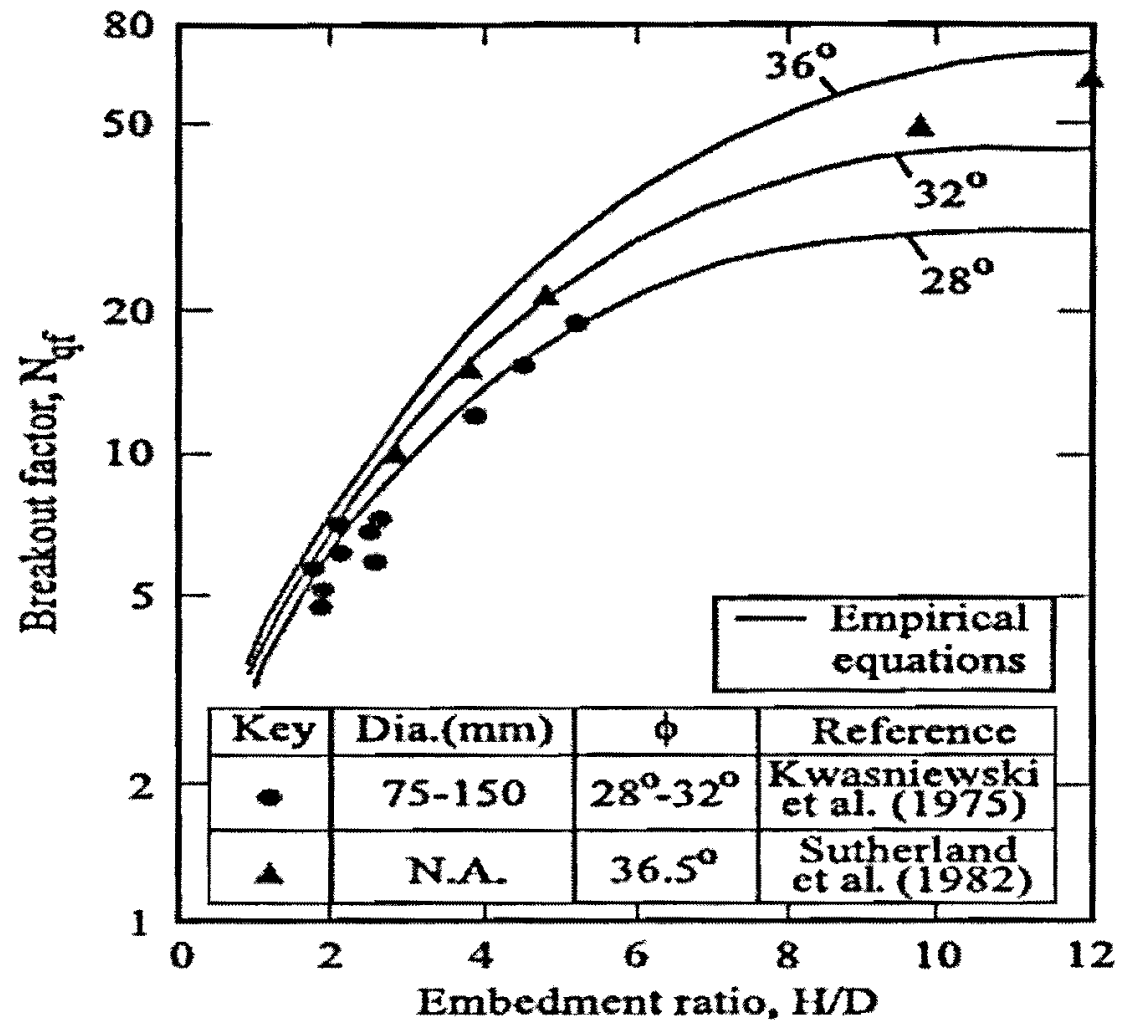

Figure 19. Comparison of breakout factors from empirical equations and previous experimental research for circular plate anchors in loose to medium-dense sand (Ilamparuthi et. al., 2002)

Good agreement between the experimental breakout factors and empirical equations is obtained (Figure 20) with five results deviating by $25 \%$ for medium dense to dense sand. Twenty percent of the test results compare fairly well and are within a range of variation of $10-20 \%$. The remaining $70 \%$ of the test results compare well, with the deviation being less than $10 \%$. It is therefore suggested that they could be applied with some confidence to predict breakout factors of circular plate anchors in dense sand. Results by Mitsch and Clemence (1985), from tests on helical anchors, however, are considerably lower than those predicted by the empirical Equations (2)-(8). This is attributed to sand disturbance during installation for this type of anchor. 


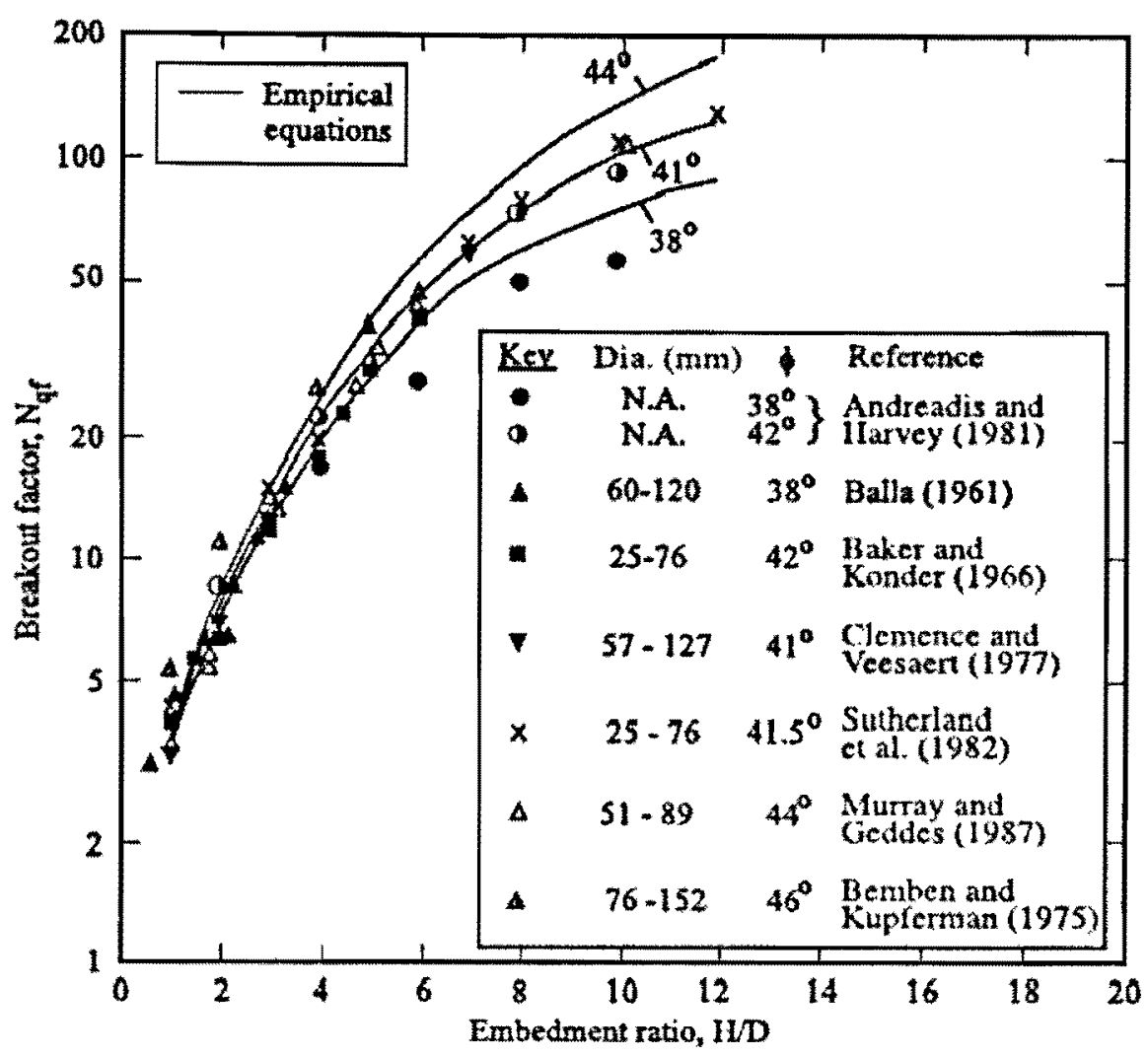

Figure 20. Comparison of breakout factors from empirical equations and previous experimental research for circular plate anchors in medium-dense to dense sand (Ilamparuthi et. al., 2002)

The variation of displacement at peak pullout load , $\delta$, with H/D is presented in Figure 21 for dense sand. Initially the relationship curves upwards, followed by a reduction in slope, becoming almost asymptotic at higher embedment ratios. The change in slope occurs at $H / D \cong 6.8$ for both 100 and $125 \mathrm{~mm}$ diameter anchors. Similar trends were observed for anchors in loose and medium-dense sand. Alternatively, this variation may be plotted logarithmically as shown for dense sand in Figure 22. The relationship is bilinear, and the change in slope may be assumed to occur at $(\mathrm{H} / \mathrm{D})_{\mathrm{CR}}$, giving values of $5.0,5.8$, and 6.8 for loose, medium-dense, and dense sand beds, respectively, which are very close to those obtained by other methods discussed earlier. Hence the relation between $\delta$ and H/D could also be used to distinguish shallow from deep anchor behavior. 


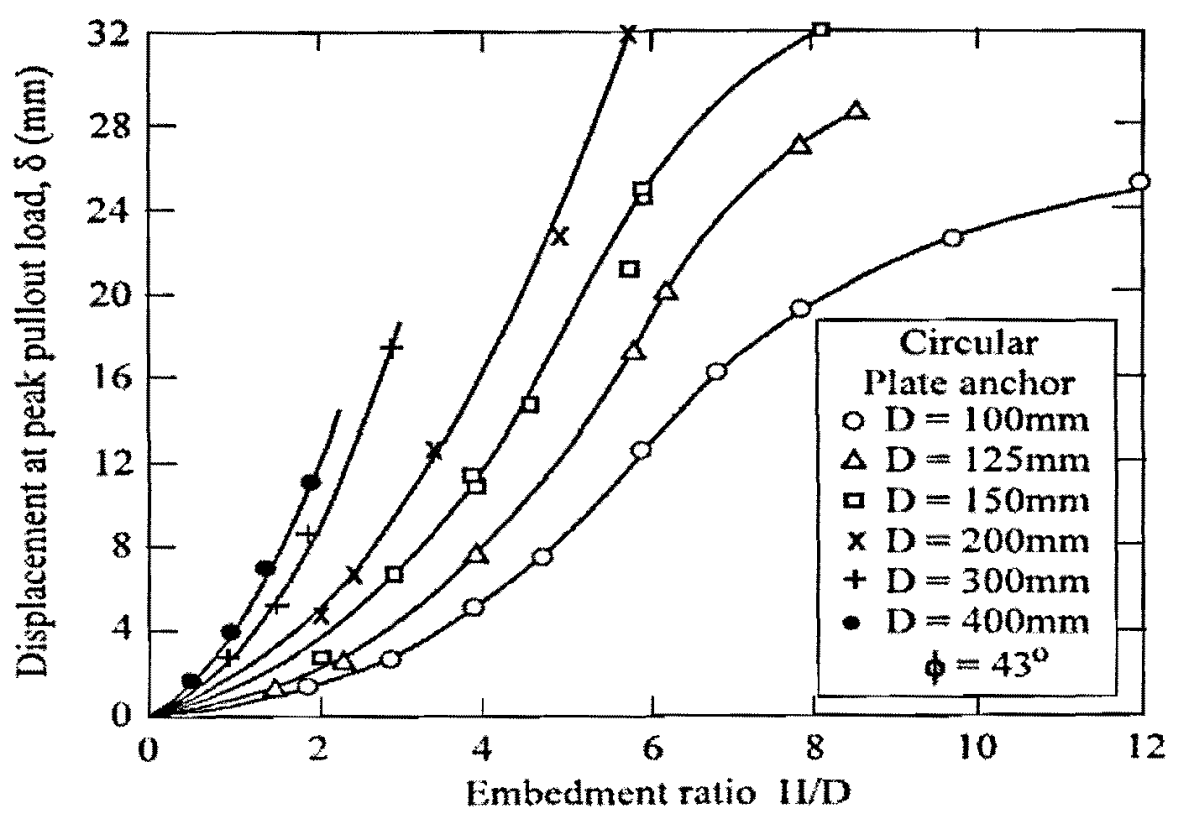

Figure 21. Displacement at peak pullout load for circular plate anchors at various embedment ratios in dense sand (Ilamparuthi et. al., 2002)

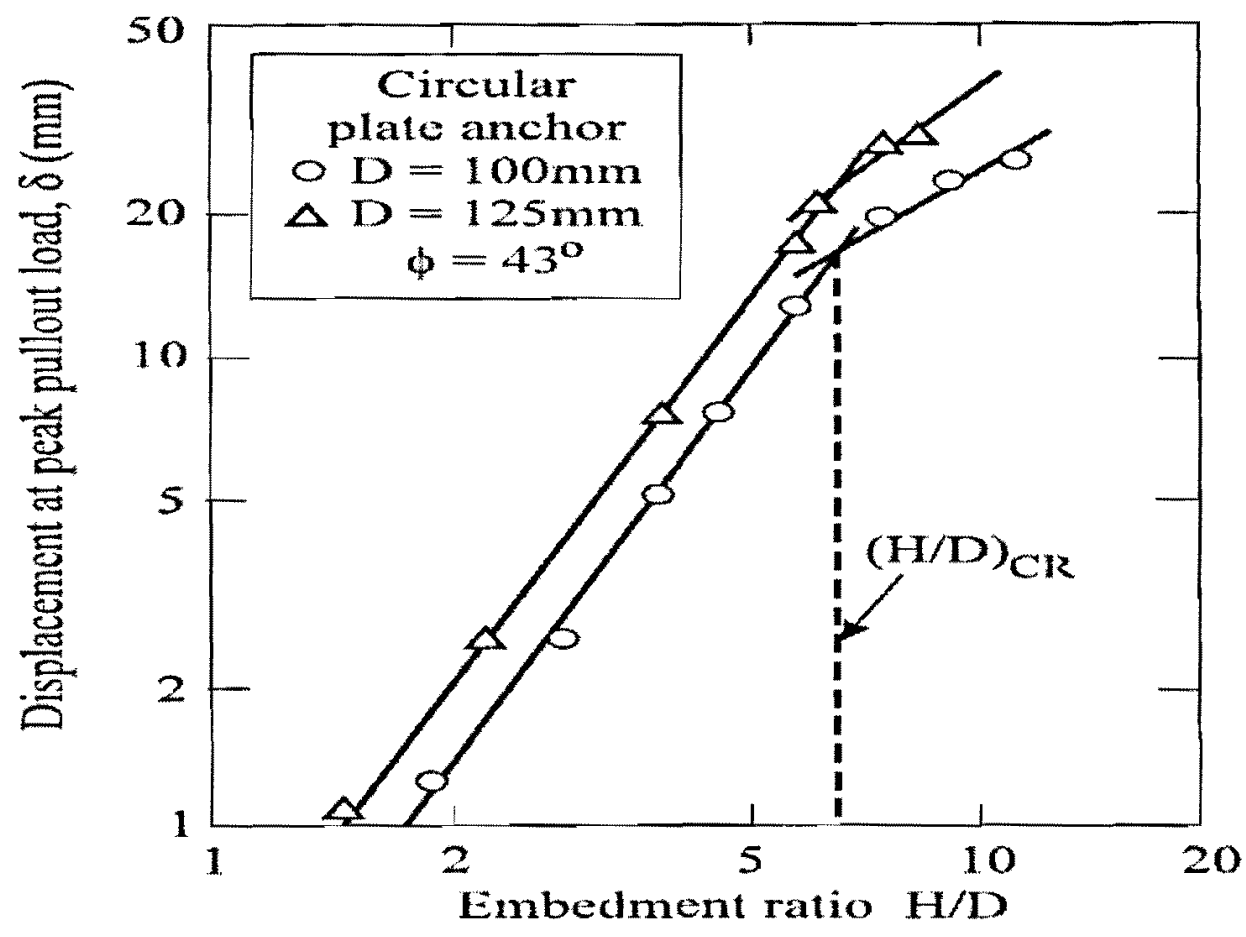

Figure 22. Determination of critical embedment ratio for circular plate anchors in dense sand (Ilamparuthi et. al., 2002) 
Knowledge of the critical embedment ratio enables anchor behavior to be estimated by the appropriate design method. Table 2 shows the critical embedment ratios obtained for loose, medium-dense, and dense sand beds from the alternative methods adopted in this research. The methods that give the more reliable values are plotted in Figure 23 as a function of $\phi$ to facilitate interpolation for other friction angles. The suggested values are 4.8,5.9, and 6.8 , respectively, for the loose, medium-dense, and dense sands studied. $(\mathrm{H} / \mathrm{D})_{\mathrm{CR}}$ values reported by other investigators are presented in Table 3. Results obtained by Baker and Kondner (1966), for $\phi=42^{\circ}$ is in good agreement with the present study, whereas the value of Vesic (1971), for dense sand is about $50 \%$ higher. The values of Meyerhof and Adams (1968), are in good agreement for loose and mediumdense sands but are about 10-20\% higher for dense sand.

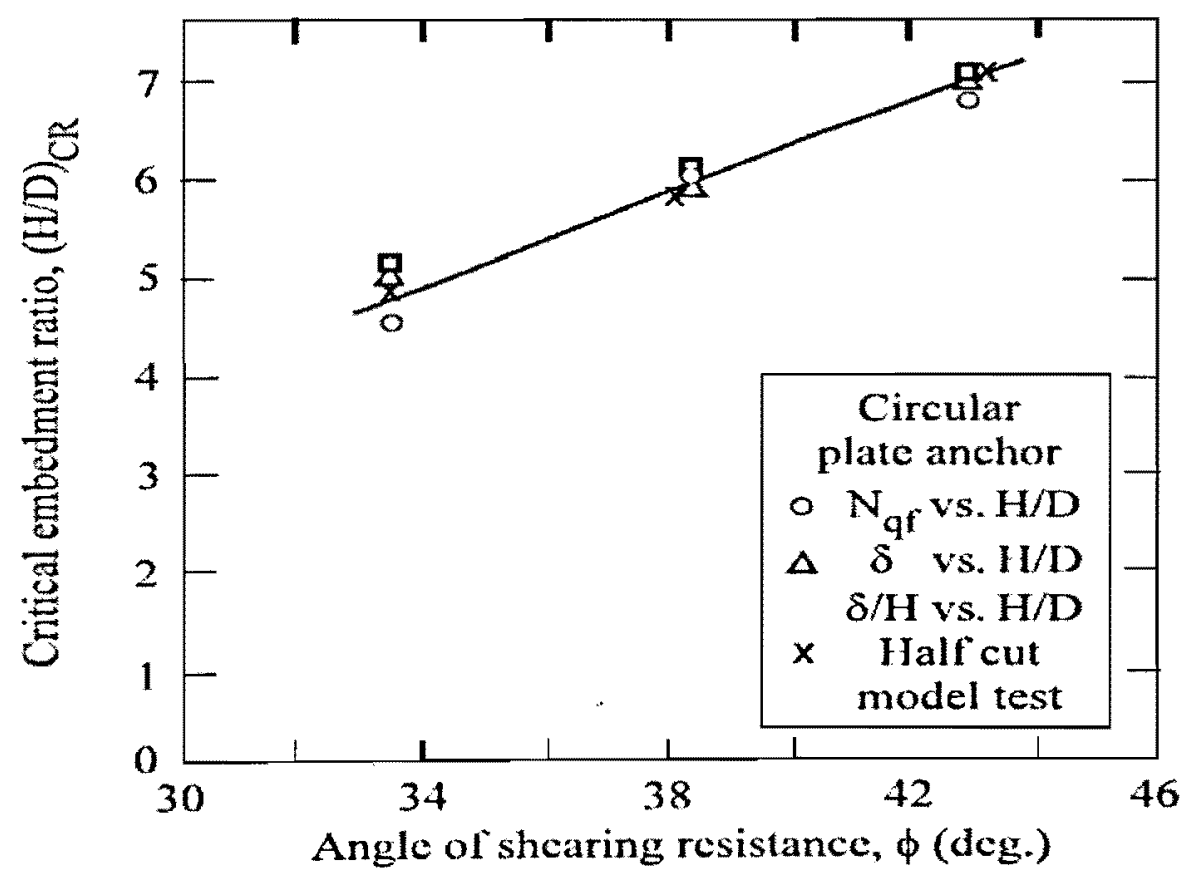

Figure 23. Comparison of alternative estimations of critical embedment ratio for circular plate anchors in sand (Ilamparuthi et. al., 2002) 
Table 2. Critical embedment ratios for circular plate anchors determined by various methods (Ilamparuthi et. al., 2002).

\begin{tabular}{|c|c|c|c|}
\hline \multirow{2}{*}{ Means of determination } & \multicolumn{3}{|c|}{ Critical embedment ratio } \\
\cline { 2 - 4 } & $\phi=33.5^{\circ}$ & $\phi=38.5^{\circ}$ & $\phi=43.0^{\circ}$ \\
\hline Load vs displacement & 4.0 & 5.9 & 6.3 \\
\hline $\mathrm{N}_{\mathrm{qf}}$ vs. H/D & 4.5 & 5.5 & 6.6 \\
\hline$\delta / \mathrm{vs}$ H/D & 5.0 & 5.8 & 6.8 \\
\hline$\delta / \mathrm{H}$ vs. H/D & 5.1 & 5.9 & 6.9 \\
\hline Surface heave & 4.0 & 5.9 & $>6.0$ \\
\hline Rupture surface in half cut model test & 4.9 & 5.8 & 6.9 \\
\hline
\end{tabular}

The values of Clemence and Veesaert (1977), are about $21 \%$ lower than those found here, and Sutherland et. al., (1982), propose comparable values for loose sand but relatively higher values for medium-dense and dense sands by about 44 and 62\%, respectively. Tagaya et. al., (1988), suggest values 36 and $21 \%$ higher than those of the authors for loose and dense sands, respectively. Clearly there is considerable disparity which may be attributed to the size of models tested, the stress levels, and the methods adopted for determining the critical depth. The sizes of models tested by most investigators were rather small, and this would certainly influence the behavior considerably. 
Table 3. Comparison of critical embedment ratios obtained from the Ilamparuthi's investigation with those from published results (llamparuthi et. al., 2002).

\begin{tabular}{|c|c|c|c|}
\hline \multirow{2}{*}{ Authors } & $\begin{array}{c}\text { Angle of } \\
\text { shearing } \\
\text { resistance, } \phi \text { ( })\end{array}$ & \multicolumn{2}{|c|}{ Critical embedment ratio } \\
\cline { 3 - 4 } & 42 & 6 & $\begin{array}{c}\text { Ilamparuthi's } \\
\text { investigation }\end{array}$ \\
\hline Baker and Kondner 1966 & Loose & 3 & 6.6 \\
\hline Vesic 1971 & Dense & 10 & 4.8 \\
\hline \multirow{3}{*}{ Meyerhof and Adams 1968 } & 30 & 4 & 6.8 \\
\cline { 2 - 4 } & 35 & 5 & 4.0 \\
\cline { 2 - 4 } & 40 & 7 & 5.1 \\
\cline { 2 - 4 } & 45 & 9 & 6.2 \\
\hline Clemence and Veesaert 1977 & 41 & 5 & 7.5 \\
\hline \multirow{3}{*}{ Sutherland et al. 1982 } & 33.6 & 4.3 & 6.4 \\
\cline { 2 - 4 } & 36.5 & 7.8 & 4.8 \\
\cline { 2 - 4 } & 41.5 & 10.5 & 5.4 \\
\hline Tagaya et al. 1988 & 32 & 6 & 6.5 \\
\hline & 42 & 8 & 6.4 \\
\hline
\end{tabular}

\subsubsection{Numerical investigation}

Although the anchorage behavior can be studied by means of laboratory or field tests, the change in stress state of soil around the anchor end cannot be easily evaluated. So in order to get the better understanding of the uplift behavior of anchors, numerical analysis is performed to illustrate the yielding condition of the soil. Since our focus is only on the anchors which are embedded in sand, therefore, the findings of two papers are elaborated in the following paragraphs.

Sakai and Tanaka (Sakai \& Tanaka, 2007) performed finite element analysis using elastoplastic model in which progressive failure with shear band effect was introduced into the constitutive equations and found that the direction of shear band propagation, within a sand layer, was observed to be a function of the density alone, regardless of the position of the layer. According to their findings, in dense bed of homogenous sand, uplift resistance decreases after the maximum uplift resistance appears and hence softening occurs. In loose bed, the uplift resistance is almost constant after the maximum uplift resistance appears and no softening occurs. The inclination 
angle of primary shear band was approximately $65^{\circ}$ in dense sand bed, $75^{\circ}$ in medium bed, and $80^{\circ}$ in loose sand which shows that angle of inclination depends on the density of sand mass.

Calculated and experimental results are shown in Figure 24 which shows the uplift resistance displacement curves in loose, medium, and dense bed. These figures show that calculated maximum uplift resistance, displacement corresponding to the maximum uplift resistance, and residual uplift resistance coincide with the observed experimental results.

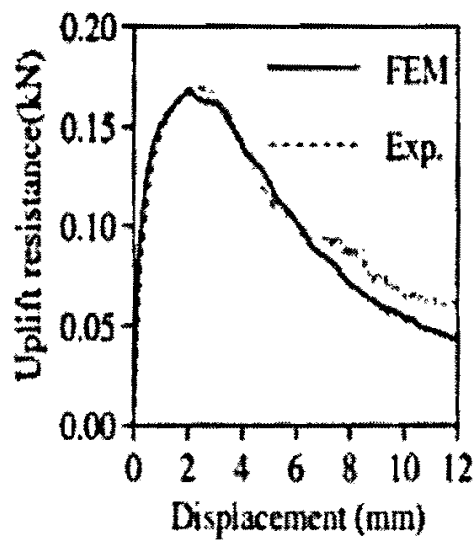

(a)

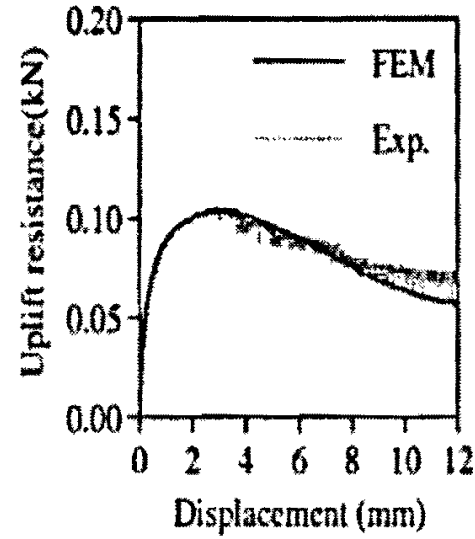

(b)

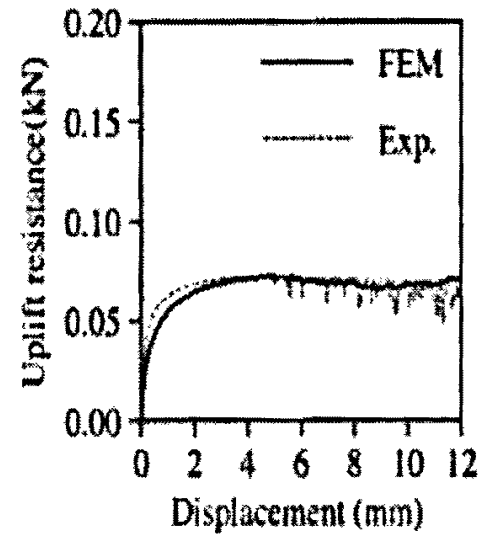

(c)

Figure 24. Uplift resistance-displacement curves in sand: (a) dense; (b) medium; (c) loose (Sakai \& Tanaka, 2007)

In dense bed, uplift resistance clearly decreases after the maximum uplift resistance appears and the softening is obvious. In loose bed, the uplift resistance is almost constant after the maximum uplift resistance appears and no softening occurs.

Hasu (Hsu et. al., 1998) performed a series of laboratory tests and numerical analysis to study the behavior of vertically embedded cylindrical anchors in sand. The load- displacement relationship was developed illustrating the yielding conditions of the sand around the anchor. They found that due to the cylindrical shape of anchor, both the shaft friction and the end resistance have substantial contribution to the pull out capacity. However, the proportions of shaft friction and end 
bearing resistance, as well as development of the pull out load-displacement relationship, may vary with the embedded depth of an anchor. If an anchor is embedded shallower than $7 \mathrm{D}$ to $8 \mathrm{D}$, a distinctive displacement softening behavior is observed for the pull out resistance of the anchor; otherwise, a displacement hardening behavior is observed. They also found that the pull out behavior of the anchor is significantly influenced by the embedded depth as shown in Figure 25.

For an anchor which is shallowly embedded (say $Z / D=4$ ) in a sand with $50 \%$ relative density, there is a significant drop off in pullout load from peak to residual as shown in Figure 25 a. In addition the shape of the shaft friction - displacement curve is very similar to that of the pullout load curve, with both peaking at virtually the same anchor displacement and then dropping off quickly to the residual state. However, the end resistance shows no peak value but approaches a constant value after the anchor has passed its peak pullout load. For an anchor embedded at a depth of 16D, the end resistance which can be mobilized is much larger than that of the shallowly embedded anchor, and it steadily increases over a displacement of $1 \mathrm{D}$, with no peak value as shown in Figure $25 \mathrm{~b}$. This is because there is sufficient volume of soil above the anchor for the end bearing zone to fully develop for a deeply embedded anchor. Meanwhile, the shaft friction peaks at small displacement and then decreases rapidly toward the residual state. Because of the way the end resistance and shaft friction develop, the pullout load increases quickly at an early loading stage, decreases slightly, and then increases steadily with increasing displacement.

Since shallowly and deeply embedded anchors show distinguished behavior in pullout load development, some critical depth can be determined to classify anchors as shallow and deep in terms of pullout load development. 

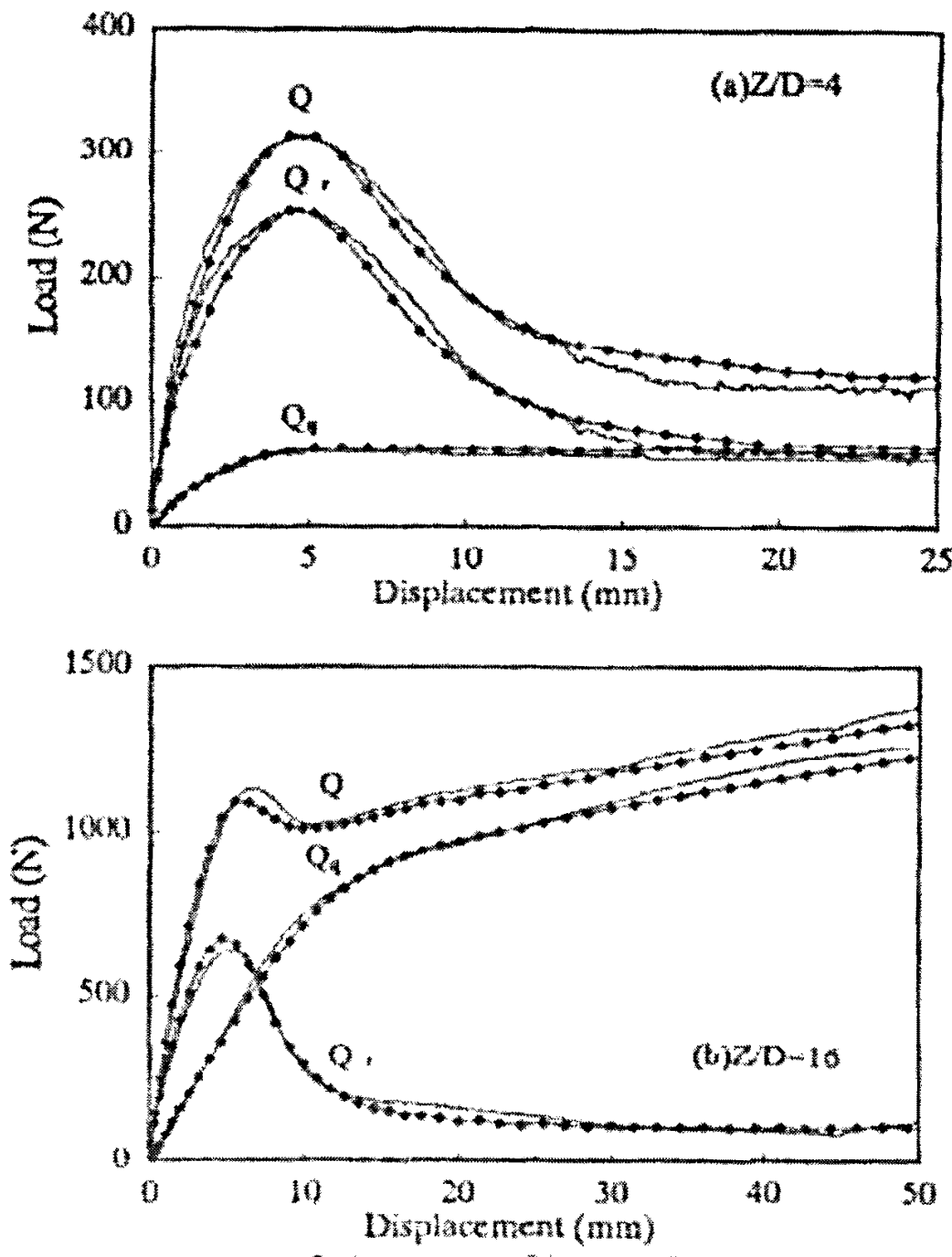

- Lab, test .. Nuricrical

Figure 25. Comparison of laboratory and numerical results on pullout load-displacement relationship at different embedded depths. $Q_{r}$, shaft resistance; $Q_{\mathbf{q}}$, end resistance (Hsu et. al., 1998)

Critical depth exists at about seven to eight times the anchor diameter (D) which differentiates the behavior of a deep anchor from shallow anchor. The size of the yielding zone which forms above an uplifting cylindrical anchor changes with embedded depth. As the embedded depth increases, say from $4 \mathrm{D}$ to $16 \mathrm{D}$, the size of the yielding zone increases from 3D to $8 \mathrm{D}$ vertically. 


\section{Chapter 3}

\section{Anchor design}

\subsection{General}

Anchor design includes an evaluation of the feasibility of using anchors, selection of an anchor system, estimates of anchor capacity, determination of embedment length. In determining the feasibility of employing anchors at a particular location, consideration shall be given to the availability or ability to obtain underground easement, proximity of buried facilities to anchor locations, and the suitability of subsurface soil and rock conditions (AASHTO, 2004). This section describes only the design method of estimating the ultimate capacity of anchors embedded in sand.

\subsection{Clemence approach}

The uplift capacity of anchors, as given in Equation la by Clemence (1985), consists of three terms: $\mathrm{W}$, the weight of the foundation and soil $\left(\mathrm{W}_{\mathrm{f}}+\mathrm{W}_{\mathrm{s}}\right)$ enclosed within the cylinder/prism shear surface; Qtu, the tip resistance developed at the anchor tip; and Qsu, the side resistance developed along the cylinder/prism shear surface.

The weight term is the easiest to evaluate because it is simply the effective weight of foundation and soil enclosed within the shear surface for drained analysis. Therefore total weights are used above the water table and submerged weights are used below the water table. The tip resistance in uplift can be provided by either suction or tension. Tip suction is an undrained phenomenon and therefore will not be acting during drained loading.

The side resistance is given by the general equation below: in which $\mathrm{D}=$ anchor depth, $\mathrm{P}=$ anchor perimeter, $\sigma_{v}^{\prime}(z)=$ vertical effective stress, $K=$ operative coefficient of horizontal soil stress $\left(\sigma_{h}^{\prime}\right.$ $\left./ \sigma_{v}^{\prime}\right), \delta^{-}=$effective stress angle of friction for the shear surface interface, $z=$ depth, $K_{0}=$ in-situ horizontal stress coefficient ( in-situ $\left.\sigma_{\mathrm{h}}^{\prime} / \sigma_{\mathrm{v}}^{\prime}\right), \mathrm{k} / \mathrm{k}_{0}=$ stress modification factor to adjust for 
construction influence, $\phi^{-}=$effective stress friction angle for the soil, and $\delta^{-} / \phi^{-}=$friction angle modification factor to adjust for interface characteristics.

$\mathrm{Qsu}=\int_{0}^{D} P(z) \sigma_{\mathrm{v}}^{\prime}(\mathrm{z}) \mathrm{K}(\mathrm{z}) \tan \delta^{-}(\mathrm{z}) \mathrm{dz}$

Qsu $=\left(k / k_{0}\right) \int_{0}^{D} P(z) \sigma_{v}^{\prime}(z) K(z) \tan \left[\phi^{-}(z)^{*} \delta^{-} / \phi^{-}\right] d z$

The anchor depth and perimeter terms are computed simply from the anchor geometry, while vertical effective stresses are computed from the effective soil unit weight and the water table location.

When it is determined that a cone or wedge breakout may develop, the side resistance will be reduced because the uplifted cone or wedge of soil will no longer be exerting a shearing resistance on the shear surface. Based on examination of the limited available data it is suggested that a reduced stress coefficient be used, which is defined by $\beta_{\mathrm{r}}=\left(2+\beta_{0}\right) / 3$ (Clemence, 1985). The reduced side resistance is then:

$\mathrm{Qsu}=\left\{\left(\mathrm{k} / \mathrm{k}_{0}\right) \int_{0}^{D} P(z) \sigma_{\mathrm{v}}^{\prime}(\mathrm{z}) \mathrm{K}(\mathrm{z}) \tan \left[\phi^{-}(\mathrm{z})^{*} \delta^{-} / \phi^{-}\right] \mathrm{dz}\right\}^{*} \beta_{\mathrm{r}} / \beta_{\mathrm{o}}$

If $\mathrm{D} / \mathrm{B}$ is larger than 5 , or $z / d$ is less than 0.25 , the reduction should be disregarded because it is minor.

The last design consideration is the upper limit or punching mechanism, defined by a bearing capacity failure. For drained loading, this is given by:

$\mathrm{Qu}(\max )=\mathrm{A}_{\mathrm{f}}\left(\mathrm{q}_{\mathrm{q}}^{-} \mathrm{N}_{\mathrm{q}} \zeta_{\mathrm{qr}} \zeta_{\mathrm{q} s} \zeta_{\mathrm{qd}}\right)+\mathrm{W}_{\mathrm{f}}+\mathrm{Qtu}$

Or in dimension less form as: 
$\left\{Q u(\max )-W_{f}^{-}-Q t u\right\} / A_{f}^{*} q_{q}^{-}=N_{q} \zeta_{q r} \zeta_{q s} \zeta_{q d}$

in which $\mathrm{Qu}(\max )=$ maximum uplifted load limited by punching, $\mathrm{A}_{\mathrm{f}}=$ foundation area, $\mathrm{q}_{\mathrm{q}}^{-}=$ effective surcharge $(\gamma \mathrm{D})$ on $\mathrm{A}_{\mathrm{f}}, \mathrm{W}_{\mathrm{f}}=$ effective weight of foundation alone. The $\mathrm{N}_{\mathrm{q}}$ term is bearing capacity factor given by:

$\mathrm{N}_{\mathrm{q}}=\mathrm{e}^{\pi \tan \phi^{-}} \tan ^{2}\left(45+\phi^{-} / 2\right)$

in which $\phi^{-}=$effective stress friction angle for the soil. The $\zeta$ terms are modification factors for soil rigidity $\left(\zeta_{q}\right)$, anchor shape $\left(\zeta_{q q}\right)$, anchor depth $\left(\zeta_{q d}\right)$, as given below:

$\left.\zeta_{\mathrm{qr}}=\exp \left\{[-4.4+0.6 \mathrm{~B} / \mathrm{L}) \tan \phi^{-}\right]+\left[\left(3.07 \sin \phi^{-}\right)\left(\log _{10} 2 \mathrm{I}_{\mathrm{r}}\right) /\left(1+\phi^{\top}\right)\right]\right\}(\leq 1.0)$

$\zeta_{\mathrm{qs}}=1+(\mathrm{B} / \mathrm{L}) \tan \phi^{-}$

$\zeta_{\text {qd }}=1+2 \tan \phi^{-}\left(1-\sin \phi^{-}\right)^{2} \tan ^{-1}(D / B)$

(with $\tan ^{-1}$ term in radians)

Where $\mathrm{D}=$ anchor depth, $\mathrm{B}=$ anchor width, $\mathrm{L}=$ anchor length, and $\mathrm{I}_{\mathrm{r}}=$ soil rigidity index given by:

$\mathrm{I}_{\mathrm{r}}=\mathrm{G} / \mathrm{q}_{\mathrm{j}}^{-} \tan \phi^{-}=\mathrm{E} / 2(1+v)^{*} 1 / \mathrm{q}_{\mathrm{j}}^{-} \tan \phi^{-}$

In which $G=$ shear modulus, $E=$ elastic modulus, $v=$ poisson's ratio, and $q_{j}^{-}=$effective surcharge $\left(\gamma^{-} \mathrm{Z}\right)$ at a height of $\mathrm{B} / 2$ above the anchor tip.

Figure 26 shows the dimensionless bearing capacity term in equation 13 for a circular or square anchor $(B=L)$, with limiting $D / B$ ratios of 0 and 5 . Increasing $D / B$ from 5 to infinity only increases the results by 2 to 4.5 percent, so the value at 5 can be conservatively when D/B is greater than 5 . 
SHALLOW SORL ANCंHORS

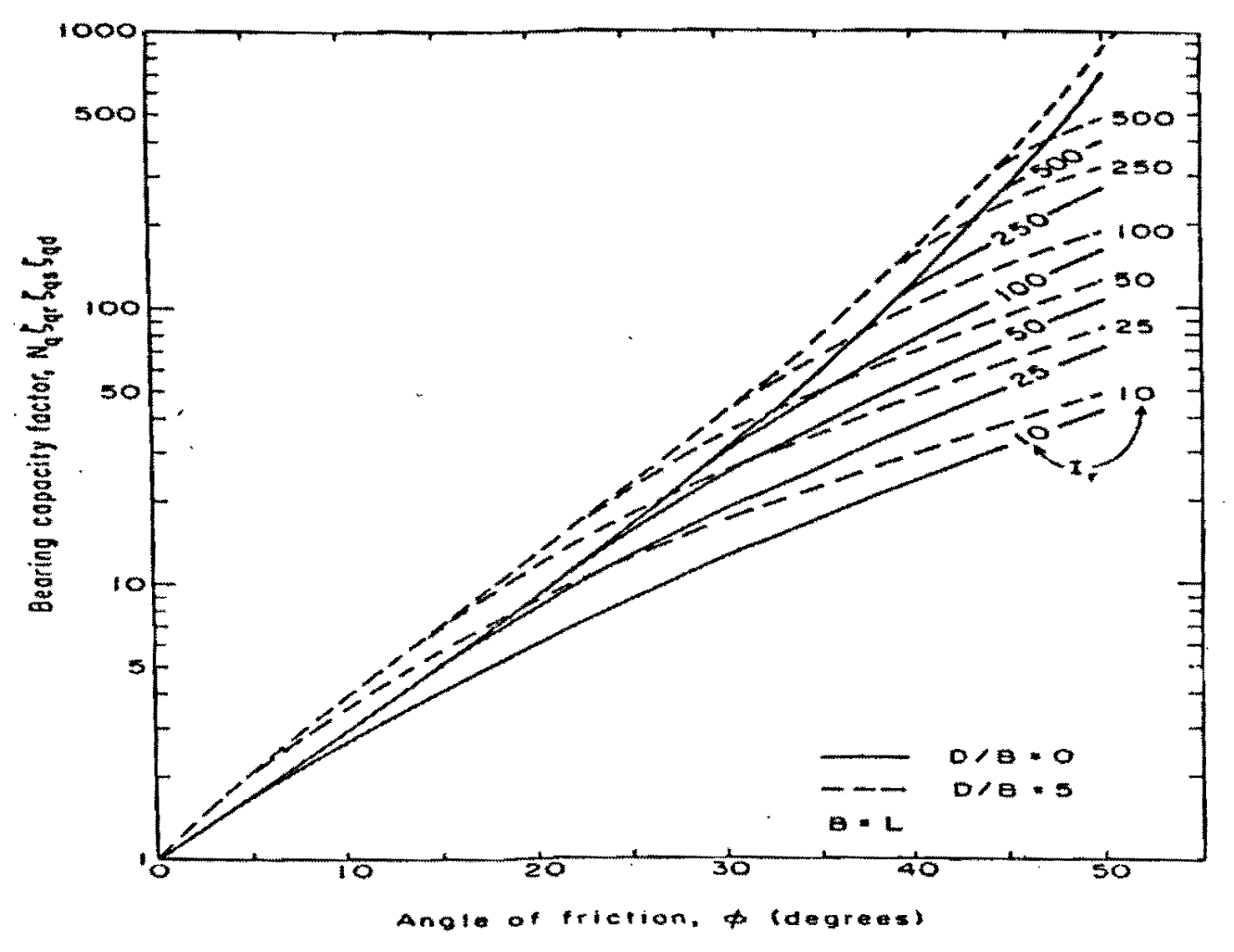

Figure 26. Modified $N_{q}$ bearing capacity factor (Clemence, 1985)

\subsection{Meyerhof and Adam approach}

Meyerhof (Meyerhof et. al., 1968) developed an approximate general theory of uplift resistance based on observations and test data presented. Because of the complex form of the failure surfaces, simplifying assumptions in respect to the actual failure surfaces are made. The theory is derived for strip or continuous footing and is then modified for use in sand and clays for circular and rectangular footings. 


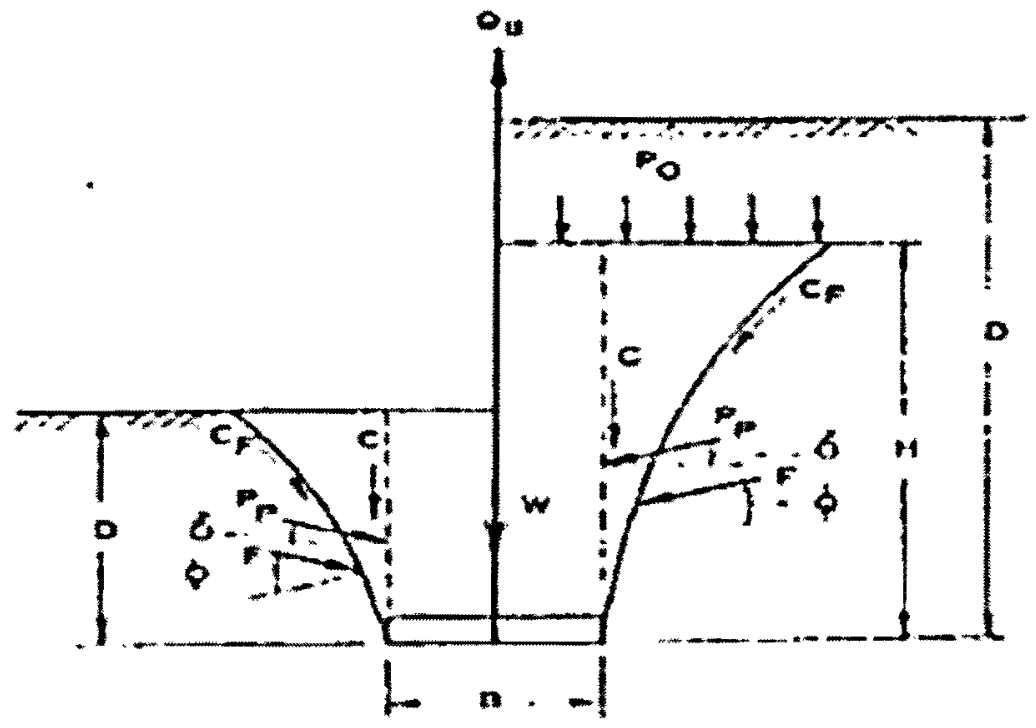

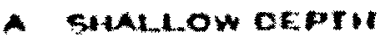

n CHEAT ORPTII

Figure 27. Failure of soil above a strip footing under uplift load (Meyerhof et. al., 1968)

\subsubsection{Strip footing:}

(a) Shallow depth:

At the ultimate uplift load $\mathrm{Qu}$ a soil mass having an approximately truncated pyramidal shape is lifted up and, for shallow footing depths, the failure surface reaches the ground surface (Figure 27a). Accordingly, a state of general shear failure exists along the failure surface on which a cohesive force $\mathrm{C}_{\mathrm{f}}$ and friction force $\mathrm{F}$ are mobilized based on a unit shearing resistance.

$\tau_{\mathrm{f}=\mathrm{C}}+\sigma \tan \phi$

where $\mathrm{C}=$ unit cohesion, $\sigma=$ normal stress on failure surface, and $\phi=$ angle of internal friction of soil. The ultimate load per unit length of footing may then be expressed by:

$\mathrm{Qu}=2 \mathrm{C}_{\mathrm{f}} \cos \alpha+2 \mathrm{~F} \cos \beta+\mathrm{W}$ 
Where $W=$ weight of lifted soil and weight of footing, and $\alpha$ and $\beta$ are average inclination with vertical of forces $C_{f}$ and $F$, respectively. In the absence of a rigorous solution for the stresses on the failure surface, it may be assumed that $\mathrm{Qu}$ is approximately given by:

$\mathrm{Qu}=2 \mathrm{C}+2 \mathrm{Pp} \sin \delta+\mathrm{W}$

Where $\mathrm{C}=\mathrm{Cd}=$ cohesion along vertical plane through footing edge and $\mathrm{Pp}=$ total passive earth pressure inclined at average angle $\delta$ acting downward on vertical plane through footing edge.

Expressing the normal component of Pp:

$\mathrm{Pp} \cos \delta=\gamma\left(\mathrm{D}^{2} / 2\right) \mathrm{K}_{\mathrm{p}}$

Where $k_{p}=$ coefficient of passive earth pressure and $\gamma=$ unit weight of soil, and substituting in to equation (21)

$Q u=2 C d+\gamma D^{2} K_{p v}+W$

Where $\mathrm{K}_{\mathrm{pv}}=\mathrm{K}_{\mathrm{P}} \tan \delta$

Test results on model strip footings indicate that, for sands, the average angle of the failure surface with the vertical varies between about $\phi / 3$ and $2 \phi / 3$. For an average value of about $\phi / 2$ for this angle, trial; calculations have shown that $\delta$ is approximately $2 \phi / 3$. From the corresponding passive earth pressure coefficient $K_{p}$ based on curved surface failure (Caquot \& Kerisel, 1949), the vertical component $\mathrm{K}_{\mathrm{pv}}$ governing the uplift resistance has been evaluated and is shown in Figure 28 . 


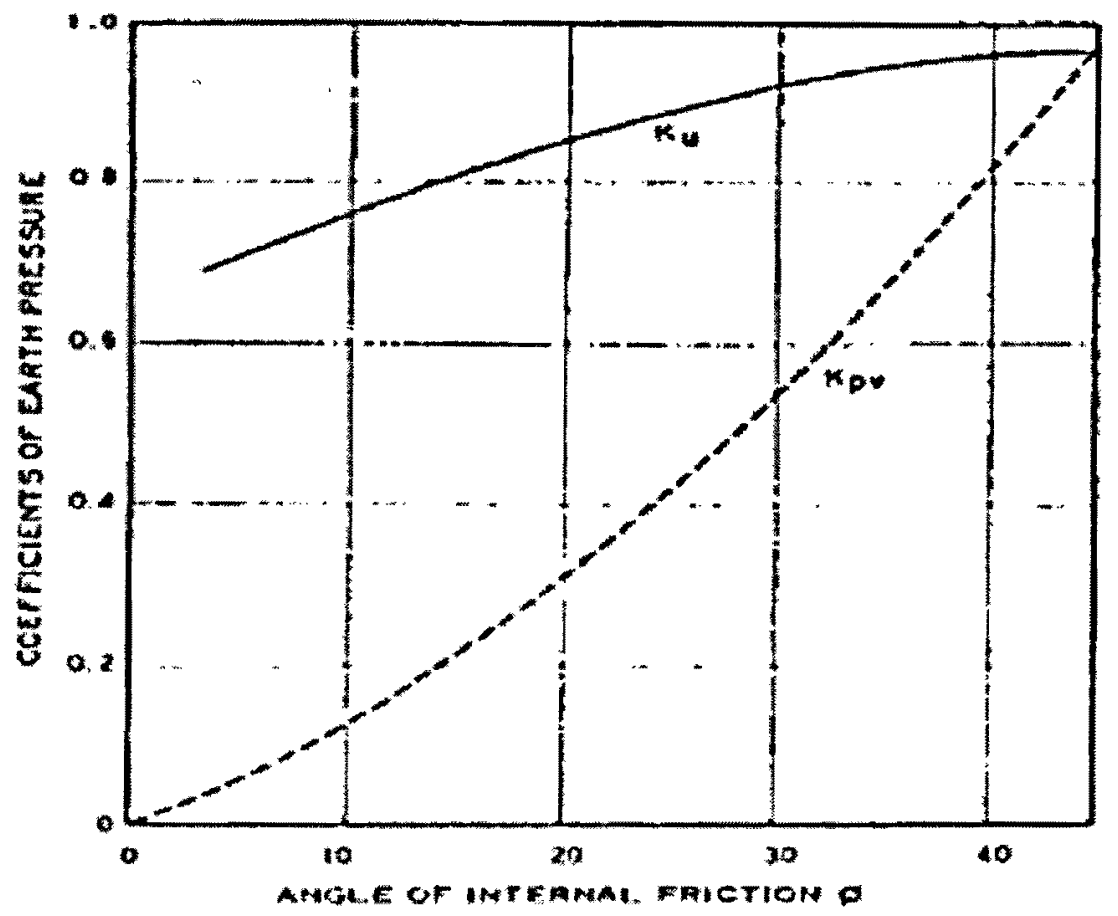

Figure 28. Theoratical uplift coefficients of earth pressure for strip footing (Meyerhof et. al., 1968)

For a given value of $\phi$, the value of $K_{p v}$ is not very sensitive of $\delta$ in the range of about $\phi / 2$ and $3 \phi / 4$, therefore for the convenience of the analysis $\mathrm{K}_{\mathrm{pv}}$ may be expressed by:

$\mathrm{K}_{\mathrm{pv}}=\mathrm{K}_{\mathrm{u}} \tan \phi$

Where $\mathrm{K}_{\mathrm{u}}=$ nominal uplift coefficient of earth pressure on vertical plane through footing edge. Thus equation (23) becomes:

$\mathrm{Qu}=2 \mathrm{Cd}+\gamma \mathrm{D}^{2} \mathrm{~K}_{\mathrm{u}} \tan \phi+\mathrm{W}$

The corresponding theoretical values of $K_{u}$ are shown in Figure 28 and are found to vary from about 0.7 to nearly unity. 


\section{(b) Great depth:}

For deep foundations failure surface does not reach the ground surface (Figure 27b). The extent of this local shear failure may be included in the analysis by limiting the vertical extent $\mathrm{H}$ of the failure surface and utilizing the surcharge pressure above the level of the failure surface as shown in equation (26).

$\mathrm{P}_{0}=\gamma(\mathrm{D}-\mathrm{H})$

On this basis equation (25) may be modified for great footing depths as:

$\mathrm{Qu}=2 \mathrm{CH}+\gamma(2 \mathrm{D}-\mathrm{H}) \mathrm{H} \mathrm{K} \mathrm{u}_{\mathrm{u}} \tan \phi+\mathrm{W}$

The magnitude of $\mathrm{H}$ can be determined from the observed extent of the failure and an analysis of the test results is given in table 4 .

Table 4. The value of H/B form test results (Meyerhof et. al., 1968).

\begin{tabular}{|l|l|l|l|l|l|l|l|}
\hline $\begin{array}{l}\text { Friction angle } \phi \\
()\end{array}$ & 20 & 25 & 30 & 35 & 40 & 45 & 48 \\
\hline Depth H/B & 2.5 & 3 & 4 & 5 & 7 & 9 & 11 \\
\hline
\end{tabular}

The upper limit of uplift resistance is given by the sum of the bearing capacity of the footing and the skin friction on the anchor shaft.

$\mathrm{Qu}=\mathrm{B}\left(\mathrm{cN}_{\mathrm{c}}+\gamma \mathrm{DN} \mathrm{N}_{\mathrm{q}}\right)+\mathrm{A}_{\mathrm{s}} \mathrm{f}_{\mathrm{s}}+\mathrm{W}$

Where $A_{s}=$ surface area of shaft, $f_{s}=$ average unit skin friction of soil on shaft, and $N_{c}$ and $N_{q}$ are bearing capacity factors as for a footing under downward loads. 


\subsubsection{Circular and rectangular footings}

\section{(a) Circular footing:}

The analysis for strip footings can be extended to circular footings by determining the shearing resistance from cohesion and passive earth pressure inclined at $\delta$ on a vertical cylindrical surface through the footing edge (Figure 3). Thus for shallow depths $(\mathrm{D}<\mathrm{H})$, equations (21) and (25) becomes

$\mathrm{Qu}=\pi \mathrm{BC}+\mathrm{s} \pi \mathrm{BP} \mathrm{P}_{\mathrm{p}} \sin \delta+\mathrm{W}$

or

$\mathrm{Qu}=\pi \mathrm{cBD}+\mathrm{s}(\pi / 2) \gamma \mathrm{BD}^{2} \mathrm{~K}_{\mathrm{u}} \tan \phi+\mathrm{W}$

Where $s=$ shape factor governing the passive earth pressure on a convex cylindrical wall. Similarly for great depths (D>H), equation (30) becomes

$\mathrm{Qu}=\pi \mathrm{cBH}+\mathrm{s}(\pi / 2) \gamma \mathrm{B}(2 \mathrm{D}-\mathrm{H}) \mathrm{HK}_{\mathrm{u}} \tan \phi+\mathrm{W}$

With an upper limit, as for the bearing capacity of a footing under downward loads, equation (28) can be used.

Test results on model circular footings reviewed below have shown that for sands the average angle of the failure surface with the vertical varies between about $\phi / 4$ and $\phi / 2$. For an average value of about $\phi / 3$, the angle $\delta$ is approximately $2 \phi / 3$ and the corresponding values of the shape factor $\mathrm{s}$ can be estimated from approximate earth pressure theories based on plane failure surfaces. For small ratios of footing depth to width of $\mathrm{D} / \mathrm{B}$ the theoretical results can be approximately represented by: 
$\mathrm{s}=1+\mathrm{mD} / \mathrm{B}$

with a maximum of:

$\mathrm{s}=1+\mathrm{mH} / \mathrm{B}$

where $\mathrm{H} / \mathrm{B}$ is given in table 4 and the coefficient $\mathrm{m}$ has the values given in table 5 .

Table 5. Values of shape factor ' $s$ ' and coefficient $m$ against friction angle (Meyerhof et. al., 1968).

\begin{tabular}{|c|c|c|c|c|c|c|c|}
\hline $\begin{array}{c}\text { Friction angle } \\
\phi(\mathrm{O})\end{array}$ & 20 & 25 & 30 & 35 & 40 & 45 & 48 \\
\hline Coefficient $\mathrm{m}$ & 0.05 & 0.1 & 0.15 & 0.25 & 0.35 & 0.5 & 0.6 \\
\hline Max factor $\mathrm{s}$ & 1.12 & 1.3 & 1.60 & 2.25 & 3.45 & 5.50 & 7.60 \\
\hline
\end{tabular}

The corresponding earth pressure coefficients designated as $\mathrm{sK}_{\mathrm{u}}$ are shown in Figure 29.

\section{(b) Rectangular footing:}

An approximate analysis for the ultimate uplift load of a rectangular footing of width B and length $\mathrm{L}$ can be obtained by assuming that the earth pressure along the perimeter of the two end portions of length $B / 2$ is governed by the shape factor $\mathrm{s}$ as for circular footings, while the passive earth pressure along the central portion of length L-B is the same as for a strip footing. On this basis it can be shown that for shallow depths:

$\mathrm{Qu}=2 \mathrm{cD}(\mathrm{B}+\mathrm{L})+\gamma \mathrm{D}^{2}(2 \mathrm{sB}+\mathrm{L}-\mathrm{B}) \mathrm{K}_{\mathrm{u}} \tan \phi+\mathrm{W}$

While for great depths:

$$
\mathrm{Qu}=2 \mathrm{cH}(\mathrm{B}+\mathrm{L})+\gamma(2 \mathrm{D}-\mathrm{H}) \mathrm{H}(2 \mathrm{Sb}+\mathrm{L}-\mathrm{B}) \mathrm{K}_{\mathrm{u}} \tan \phi+\mathrm{W}
$$

For square footings $\mathrm{B}=\mathrm{L}$ in the above expressions. 


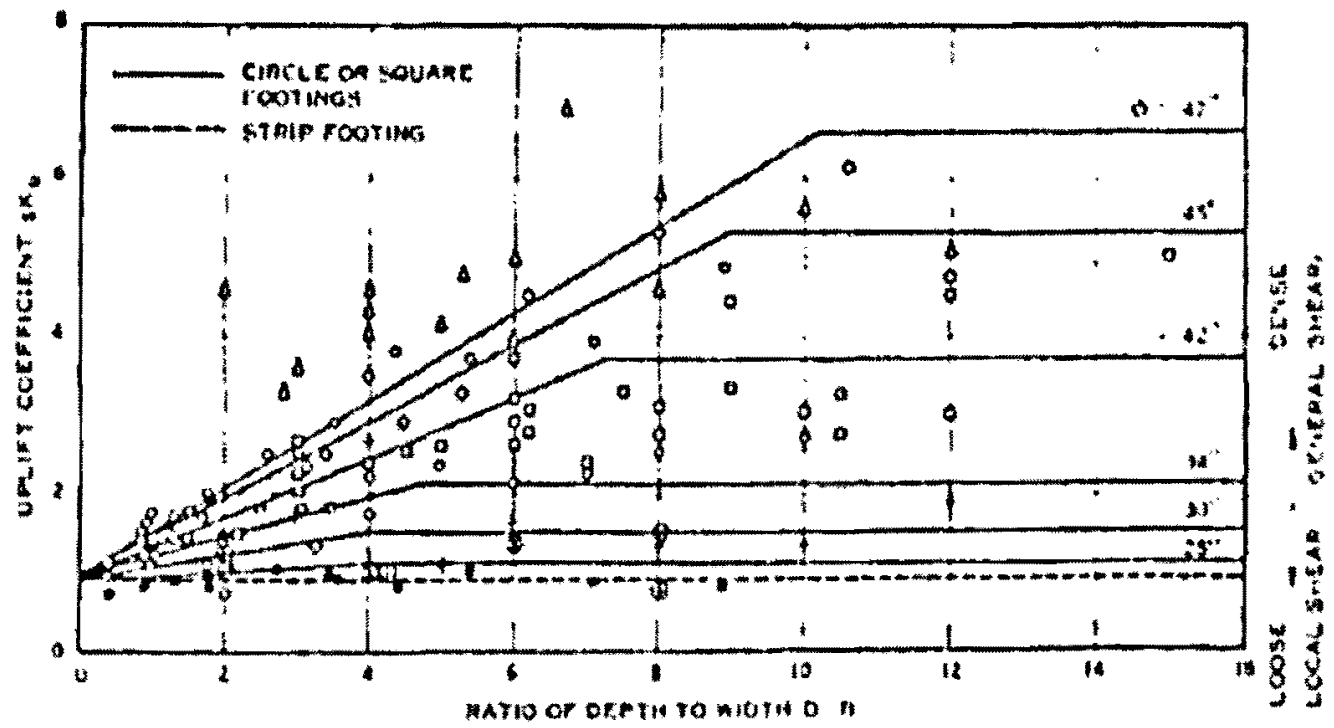

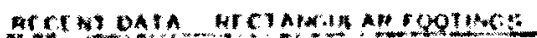

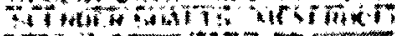

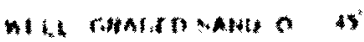

arwat ton:

c) $(\cos ) * 1^{*}$

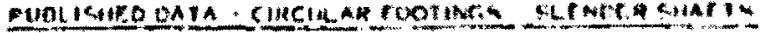

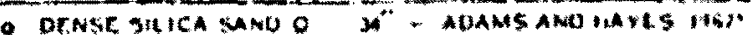

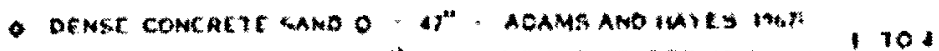

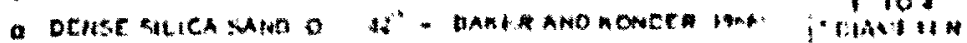

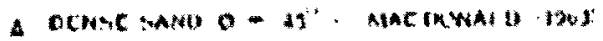

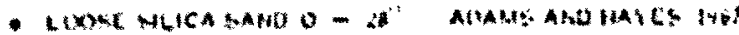

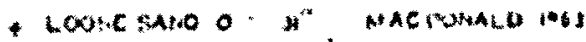

Figure 29. Comparison of theory and model tests for footings in sand (Meyerhof et. al., 1968) 


\section{Chapter 4}

\section{Codes for anchors}

\subsection{General}

Association of State Highway and Transportation Officials (AASHTO), Euro Code 7, and US Army corps of Engineers have published some code for straight shaft and grouted anchors without plates. There is no code available which provides some guidance to calculate the ultimate capacity of anchor plates. US Army Corps of Engineers have published some codes for straight shaft anchors without plates to calculate ultimate capacity and AASHTO has provided some guidelines for preliminary design of straight shaft anchors without plates but none of them talk about plate anchors. Euro code also gives some guidelines about straight shaft anchors and recommends that design should be based on testing results. Section 4.1 discusses about Euro code 7 .

\subsection{Euro Code 7}

Section 8 of Euro code 7, EN 1997-1, (Andrew et. al., 2008) applies to prestressed anchors without anchor plates with scant attention to anchorages with plates. No guidance is given in Euro code 7 on how to design anchorages by calculations and also there is no code available for anchors with plates. Euro code recommends that designs are based on testing results. According to Euro code 7 the design pull-out resistance of an anchorage $\mathrm{R}_{\mathrm{a}, \mathrm{d}}$ must satisfy the following condition:

$\mathrm{P}_{\mathrm{d}} \leq \mathrm{R}_{\mathrm{a}, \mathrm{d}}$

Where $P_{d}$ is the design load in the anchorage, i.e. the larger of the anchor force derived from an ultimate limit state verification of the retained structure ( $\mathrm{P}_{U L S}$ ) and that derived from a serviceability limit state verification $\left(\mathrm{P}_{\mathrm{SLSS}}\right)$, i.e.:

$\mathrm{P}_{\mathrm{d}}=\max \left(\mathrm{P}_{\mathrm{ULS}}, \mathrm{P}_{\mathrm{SLS}}\right)$ 
There is some debate as to whether the anchor force that is derived from an ultimate limit state (ULS) verification of an anchored retaining wall (i.e. $P_{U L S}$ ) is the largest force the anchor will have to withstand. At the ULS, earth pressure on the back of the wall approach limiting active $\left(\mathrm{K}_{\mathrm{a}}\right)$ values and those on the front limiting passive $\left(\mathrm{K}_{\mathrm{p}}\right)$ values.

In some circumstances, it is possible for the serviceability limit state (SLS) force, $\mathrm{P}_{\mathrm{SLS}}$, to be of similar magnitude to-or even larger than- $P_{U L S}$. At the SLS, earth pressures on the back of the wall may remain close to their in situ (i.e. $\mathrm{K}_{0}$ ) values, particularly for inflexible walls in stiff soils. Since values of $K_{o}$ can be considerably larger than $K_{a}$, it is possible for $P_{U L S}$ and $P_{s L S}$ to be of the same magnitude. The value of $\mathrm{P}_{\mathrm{ULS}}$ obtained from ultimate limit state calculations of wall stability includes a load factor $\gamma_{G}$, which is equal to 1.35 in design approach. Therefore it is recommended to multiply $P_{S L S}$ by a model factor $\gamma_{R d}=\gamma_{G}=1.35$. The design load in the anchorage $P_{d}$ is then:

$P_{d}=\max \left(P_{U L S}, \gamma_{R d} P_{S L S}\right)$

Euro code 7 discusses only the design pull out resistance $R_{a, d}$ from the result of tests but does not offer any mathematical formula to calculate design pull out load.

Euro code has specific requirements regarding anchorage tests. Unless their performance and durability can be demonstrated by (documented) successful comparable experience, anchorage systems must be verified by investigation tests. The characteristic pull out resistance $R_{a, k}$ of grouted and screw anchorages must be determined from suitability tests, and all grouted anchorages must undergo acceptance tests. Suitability tests are not intended to determine the characteristic pull out resistance. Their purpose is to prove that the anchorages are suitable for the conditions on the site. The important distinction is that the loading in a suitability test does not exceed the proof load. 


\subsubsection{Anchorage tests}

Euro code 7 discusses three types of anchorage tests: investigation, suitability, and acceptance test.

\section{Investigation tests}

An investigation test is a load test to establish the ultimate resistance of an anchor at the grout/ground interface and to determine the characteristics of the anchorage in the working load range. Investigation tests are performed, before working anchorages are installed, to establish the anchorage's ultimate pull out resistance in the ground conditions at the site.

In investigation tests, the anchorage should either be loaded to its failure load $R_{a}$ or to a proof load $P_{p}$, which must be limited to (for test method 1 and 2) the smaller of:

$\mathrm{P}_{\mathrm{p}} \leq 0.8 \mathrm{P}_{\mathrm{t}, \mathrm{k}}$ and $\mathrm{P}_{\mathrm{p}} \leq 0.8 \mathrm{P}_{\mathrm{t} 0.1, \mathrm{k}}$

Or (for test method 3) the smaller of:

$\mathrm{P}_{\mathrm{p}} \leq 0.8 \mathrm{P}_{\mathrm{t}, \mathrm{k}}$ and $\mathrm{P}_{\mathrm{p}} \leq 0.9 \mathrm{P}_{\mathrm{t} 0.1, \mathrm{k}}$

Where $P_{t, k}$ is the tendon's characteristic tensile load capacity and $P_{t 0.1, k}$ is the characteristic tensile load at $0.1 \%$ strain.

In test method ' 1 ' and ' 2 ' load is applied in incremental cycles but in test method ' 1 ' load is applied to a maximum test load and in test method ' 2 ' load is applied either to a maximum test load or up to failure. In test method ' 3 ' load is applied in incremental steps to a maximum test load.

\section{Suitability tests}

A suitability test is a load test on site to confirm that a particular anchor design will be adequate in particular ground conditions. Anchorages subjected to suitability tests may be used as working 
anchorages. At least three suitability tests shall be performed on anchorages constructed under identical conditions to the working anchors.

The proof load $P_{p}$ applied in suitability tests should be the greater of:

$P_{p} \geq 1.25 P_{0}$ and $P_{p} \geq 0.9 R_{d}$

Where $P_{0}$ is the lock-off load and $R_{d}$ is the required design resistance of the anchor; and must be limited to (for test methods 1 and 2):

$\mathrm{P}_{\mathrm{p}} \leq 0.95 \mathrm{P}_{\mathrm{t} 0,1, \mathrm{k}}$ or

$P_{p} \leq 0.99 P_{t 0.1, k}$

\section{Acceptance test}

An acceptance test is a load test on site to confirm that each anchorage meets the design requirements.

Acceptance tests must be carried out on all working anchorages to demonstrate that a proof load $P_{p}$ can be sustained and to determine creep or load loss characteristics under serviceability conditions.

The proof load $\mathrm{P}_{\mathrm{p}}$ applied in acceptance tests should be (for test methods 1 and 2):

$1.25 \mathrm{P}_{0} \leq \mathrm{P}_{\mathrm{p}} \leq 0.9 \mathrm{P}_{\mathrm{t} 0.1, \mathrm{k}}$

Or for test method 3:

$\mathrm{P}_{\mathrm{p}}=1.25 \mathrm{P}_{0}$ or $\mathrm{P}_{\mathrm{p}}=\mathrm{R}_{\mathrm{d}}$

Where $P_{0}$ is the lock-off load, $P_{t 0.1, k}$ is the tendon's characteristic tensile load at $0.1 \%$ strain, and $R_{d}$ is the required design resistance of the anchor. 
With the resistance factor recommended in Euro code the proof load in an acceptance test using test method 3 approach the anchor's ultimate pull out resistance, which could lead to unacceptable creep in anchors. To avoid this, it has been recommended that $P_{p}$ be further limited to:

$\mathrm{P}_{\mathrm{p}} \leq 1.15 \mathrm{P}_{\mathrm{k}, \mathrm{SLS}}$

\subsubsection{Pull-out resistance from tests}

\section{Characteristics pull-out resistance}

The characteristic pull-out resistance of a grouted anchorage is the lowest of the following:

- bond resistance between the grout and ground (external resistance, $R_{a, k}$ )

- bond resistance between the grout and tendon (internal resistance, $R_{i, k}$ )

- tensile capacity of the tendon $\left(\mathrm{P}_{\mathrm{t}, \mathrm{k}}\right)$

- capacity of the anchor head

During anchorage test, failure will occur in the weakest element. Design based on testing does not identify which failure mode is involved.

When the characteristic pull-out resistance $R_{a, k}$ is obtained from investigation tests, its value is given by:

$\mathrm{R}_{\mathrm{a}, \mathrm{k}}=\min \left(\mathrm{R}_{\mathrm{a}}, \mathrm{P}_{\mathrm{p}}\right)$

Where $R_{a}$ is the measured failure load and $P_{p}$ is the maximum proof load applied in the test.

When the characteristic pull-out resistance $R_{a, k}$ is obtained from suitability tests, its value is given by:

$\mathrm{R}_{\mathrm{a}, \mathrm{k}}=\mathrm{P}_{\mathrm{p}} / \zeta_{\mathrm{a}}$ 
Where $P_{p}$ is the measured proof load and $\zeta_{a}$ is a correlation factor that accounts for the number of suitability tests performed. Euro code provides no recommended values for $\zeta_{\mathrm{a}}$. Previous suggestions for the values of $\zeta_{\mathrm{a}}$ are summarized in the table below:

Table 6. Suggested values of $\zeta_{\mathrm{a}}$ (Andrew et. al., 2008).

\begin{tabular}{|c|c|c|c|c|}
\hline \multirow{2}{*}{ Reference } & $\zeta_{\mathrm{a}}$ on measured resistance $\left(\mathrm{R}_{\mathrm{am}}\right)$ & \multicolumn{3}{|c|}{ No. of anchorage tests } \\
\hline \multirow{2}{*}{ ENV 1997-1 } & Mean $\left(\mathrm{R}_{\mathrm{am}, \text { mean }}\right)$ & 1 & 2 & $>2$ \\
\cline { 2 - 5 } & Minimum $\left(\mathrm{R}_{\mathrm{am}, \min }\right)$ & 1.5 & 1.35 & 1.3 \\
\hline \multirow{2}{*}{ Designers' guide } & Mean $\left(\mathrm{R}_{\mathrm{am}, \text { mean }}\right)$ & 1.2 & 1.25 & 1.1 \\
\cline { 2 - 5 } & Minimum $\left(\mathrm{R}_{\mathrm{am} \text {,min }}\right)$ & 1.2 & - & 1.1 \\
\cline { 2 - 5 } & &
\end{tabular}

It is impossible to design anchorages on the basis of suitability tests until suitable values are made available in a future revision of standard.

When the characteristic pull-out resistance $\mathrm{R}_{\mathrm{a}, \mathrm{k}}$ is obtained from acceptance tests, its value is given by:

$R_{\mathrm{a}, \mathrm{k}}=\mathrm{P}_{\mathrm{p}}$

where $P_{p}$ is the measured proof load.

\section{Design pull-out resistance}

The design pull out resistance of an anchorage $R_{a, d}$ is given $b$ :

$\mathrm{R}_{\mathrm{a}, \mathrm{d}}=\mathrm{R}_{\mathrm{a}, \mathrm{k}} / \gamma_{\mathrm{a}}$

Where $R_{a, k}$ is the anchor's characteristic pull out resistance and $\gamma_{a}$ is partial factor.

EN 19697-1 suggests $\gamma_{\mathrm{a}}=1.1$ for design approach 1 and 2 and 1.0 for design approach 3 . 


\section{Chapter 5}

\section{Discussions and findings}

\subsection{Comparison of Ilamparuthi's test results with Meyerhof's design equations}

Ilamparuthi's (Ilamparuthi et. al., 2002) test data was used in Meyerhof's proposed anchor design equation (30) and results, which are shown in column 9 of table 7 , were compared with the laboratory results and were found to be comparable with the experimental results, but little bit on the lower side. The percentage difference is shown in column 10 of table 7. Good agreement exits between experimental results and the one obtained from Meyerhof's proposed design equation with only four results deviating between 20 to $25 \%$. Sixty percent of the test results compare well with a deviation between 10 to $15 \%$. Two results have only deviation of about 3 and $7 \%$. It is therefore suggested that Meyerhof's proposed design equation can be used with confidant for shallow anchor design.

Following are the details of the Ilamparuthi's test data which was used in Meyerhof's equations.

Dry unit weight of sand (Palar River sand), $\gamma_{\mathrm{d}}=14.3\left(\mathrm{KN} / \mathrm{M}^{3}\right)$

Anchor thickness $\quad=6 \mathrm{~mm}$

Mild steel anchor unit weight used to calculate weight of anchor $=76.97 \mathrm{KN} / \mathrm{M}^{3}$

S, shape factor, was calculated using equation 32 and value of ' $m$ ' was taken from table 5 . 
Table 7. Comparison of llamparuthi's test results on shallow anchors in dense sand with Meyerhof's design formula .

\begin{tabular}{|c|c|c|c|c|c|c|c|c|c|}
\hline $\begin{array}{l}\text { Dry unit } \\
\text { weight of } \\
\text { sand } \gamma_{\mathrm{d}} \\
\left(\mathrm{KN} / \mathrm{M}^{3}\right) \\
\end{array}$ & $\begin{array}{l}\text { Wut } \\
\text { Anchor } \\
\text { diameter, } \\
\mathrm{B}(\mathrm{m})\end{array}$ & $\begin{array}{l} \\
\mathrm{D} / \mathrm{B} \\
\end{array}$ & $\begin{array}{l}\text { Weight } \\
\text { of lifted } \\
\text { soil and } \\
\text { footing, } \\
W(\mathrm{KN}) \\
\end{array}$ & $\begin{array}{l}\text { Angle } \\
\text { of } \\
\text { internal } \\
\text { friction, } \\
\phi \\
\text { (Degree } \\
\end{array}$ & $\begin{array}{c}\text { Nominal } \\
\text { uplift } \\
\text { coefficie } \\
\text { nt of } \\
\text { earth } \\
\text { pressure, } \\
\text { Ku } \\
\text { (Figure } \\
28)\end{array}$ & $\begin{array}{l}\text { Shape } \\
\text { Shactor, } \\
\text { fas } \\
\text { Sy }\end{array}$ & $\begin{array}{c}\text { Peak } \\
\text { load } \\
\text { } \\
\text { lampar } \\
\text { uthi lab } \\
\text { results), } \\
Q_{f} \\
(\mathrm{KN})\end{array}$ & $\begin{array}{l}\text { Peak } \\
\text { load } \\
\text { (Calcul } \\
\text { ated } \\
\text { from } \\
\text { Meyerh } \\
\text { of } \\
\text { equatio } \\
\text { n), } Q \\
(\mathrm{KN})\end{array}$ & $\begin{array}{l}\text { Percentage } \\
\text { difference }\end{array}$ \\
\hline 14.30 & 0.10 & 0.85 & 0.01 & 43.00 & 0.95 & 1.32 & 0.039 & 0.029 & $(25.610)$ \\
\hline 14.30 & 0.10 & 1.90 & 0.03 & 43.00 & 0.95 & 1.72 & 0.181 & 0.154 & (15.113) \\
\hline 14.30 & 0.10 & 2.87 & 0.04 & 43.00 & 0.95 & 2.09 & 0.466 & 0.383 & $(17.917)$ \\
\hline 14.30 & 0.13 & 1.44 & 0.04 & 43.00 & 0.95 & 1.55 & 0.186 & 0.180 & $(3.120)$ \\
\hline 14.30 & 0.13 & 2.27 & 0.06 & 43.00 & 0.95 & 1.86 & 0.519 & 0.479 & $(7.629)$ \\
\hline 14.30 & 0.15 & 2.01 & 0.08 & 43.00 & 0.95 & 1.76 & 0.723 & 0.558 & (22.773) \\
\hline 14.30 & 0.15 & 1.98 & 0.08 & 43.00 & 0.95 & 1.75 & 0.674 & 0.541 & (19.706) \\
\hline 14.30 & 0.15 & 2.80 & 0.11 & 43.00 & 0.95 & 2.06 & 1.470 & 1.196 & (18.622) \\
\hline 14.30 & 0.20 & 2.03 & 0.20 & 43.00 & 0.95 & 1.77 & 1.804 & 1.362 & $(24.527)$ \\
\hline 14.30 & 0.20 & 2.43 & 0.23 & 43.00 & 0.95 & 1.92 & 2.353 & 2.037 & (13.421) \\
\hline 14.30 & 0.30 & 0.92 & 0.31 & 43.00 & 0.95 & 1.35 & 1.235 & 0.923 & $(25.227)$ \\
\hline 14.30 & 0.30 & 1.43 & 0.47 & 43.00 & 0.95 & 1.54 & 2.500 & 2.165 & (13.403) \\
\hline 14.30 & 0.30 & 1.88 & 0.60 & 43.00 & 0.95 & 1.71 & 4.706 & 3.854 & (18.103) \\
\hline 14.30 & 0.40 & 0.47 & 0.39 & 43.00 & 0.95 & 1.18 & 0.824 & 0.721 & $(12.449)$ \\
\hline 14.30 & 0.40 & 0.98 & 0.76 & 43.00 & 0.95 & 1.37 & 2.745 & 2.438 & $(11.190)$ \\
\hline 14.30 & 0.40 & 1.39 & 1.05 & 43.00 & 0.95 & 1.53 & 5.980 & 4.809 & $(19.589)$ \\
\hline
\end{tabular}

\subsection{Application of Euro code for plate anchors}

Section 8 of Euro code 7, EN 1997-1, (Andrew et. al., 2008) applies to prestressed anchors without anchor plates with scant attention to the anchors with plates. No guidance is given in Euro code 7 on how to design anchorages by calculations and also there is no code available for anchors with plates. Since Euro code recommends that designs are based on testing results, therefore author suggests that Euro code can be used for plate anchors with the procedure described in section 4.1. 


\subsection{Influence of sand density and H/D ratio on ultimate uplift capacity of anchors}

The peak load increases at a higher rate with increased depth of embedment for a given anchor diameter as shown in Figure 11.The peak pullout load increases with the increase in density of sand as shown in Figure 14. The break out factor increases with the increase in H/D ratio as shown in Figure 15. Peak pullout loads increase with an increase in $\mathrm{D}$ for each depth of embedment $\mathrm{H}$ for the range of diameters studied as shown in Figure 13. 


\section{Chapter 6}

\section{Conclusions}

There are mainly three distinctive failure planes used in practice for anchor design. The first failure plane is a frictional cylinder extending vertically to soil surface from the anchor edge. The second type is a truncated cone extending from the anchor with an apex angle of $90^{\circ}+\phi$, where $\phi$ is the friction angle of soil. The last one is a circular surface extending from the edge of the anchor and intersecting the ground surface with an angle of approximately $45^{\circ}-\phi / 2$.

The uplift capacity of anchors is strongly dependent on their diameter, embedment ratio, and sand density. The load displacement response is different for shallow and deep anchor conditions. For shallow circular anchor plates in sand, the load-displacement behaviour was observed to be three phase exhibiting pre peak behaviour with a rapid increase in load, post peak behaviour with a rapid load reduction, and residual behaviour associated with a moderate decrease in load at large displacements. In deep circular anchor plates in sand, a two-phase load-displacement behaviour was characterized by gradually increasing pre-peak trend followed by a very slowly decreasing residual post-peak behaviour. The critical embedment ratio increases with an increase in sand density; the recommended values are $4.8,5.9$, and 6.8 for loose, medium-dense, and dense sand, respectively for anchors in the 100-150 mm diameter range. The peak load increases at a higher rate with increased depth of embedment for a given anchor diameter. A similar, but less pronounced trend is found for anchors in loose and medium-dense sand. For a given $H$ and $D$ as friction angle increases Qf increases which shows that ultimate pull out capacity increases with the increase in density. Peak pullout loads increase linearly with an increase in D for each depth of embedment $\mathrm{H}$. 
Ilamparuthi (Ilamparuthi et. al., 2002) has formulated a set of empirical equations from which ultimate uplift capacity of circular anchors in sand can be predicted. Comparison of break out factors calculated from Ilamparuthi,s proposed empirical equations with those obtained from published test results shows that the results calculated from empirical equations are comparable with the laboratory results.

llamparuthi's test data was used in Meyerhof's proposed anchor design equation (30) and results, which are shown in column 9 of table 7 , were compared with the laboratory results and were found to be comparable with the experimental results, but little bit on the lower side. The percentage difference is shown in column 10 of table 7. Good agreement exits between experimental results and the one obtained from Meyerhof's proposed design equation with only four results deviating between 20 to $25 \%$. Sixty percent of the test results compare well with a deviation between 10 to $15 \%$. Two results have only deviation of about 3 and $7 \%$. It is therefore suggested that Meyerhof's proposed design equation can be used with confidant for shallow anchor design.

Section 8 of Euro code 7, EN 1997-1, applies to prestressed anchors without anchor plates. No guidance is given in Euro code 7 on how to design anchorages by calculations and also there is no code available for anchors with plates. Euro code recommends that designs are based on testing results. Euro code has specific requirements regarding anchorage tests. Unless their performance and durability can be demonstrated by (documented) successful comparable experience, anchorage systems must be verified by investigation tests. The characteristic pull out resistance $R_{a, k}$ of grouted and screw anchorages must be determined from suitability tests, and all grouted anchorages must undergo acceptance tests. Suitability tests are not intended to determine the characteristic pull out resistance. Their purpose is to prove that the anchorages are suitable for the conditions on the site. Since Euro code recommends that designs are based on testing results, 
therefore author suggests that Euro code can be used for plate anchors with the procedure described in section 4.1. The characteristic pull out resistance $R_{a, k}$ for plate anchors may be determined from suitability tests. 
Sakai, T., and T. Tanaka (2007). Experimental and numerical study of uplift behavior of shallow circular anchor in two-layered sand. Journal of Geotechnical and Geoenvironmental Engineering, 133(4): 469-477.

S. T. Hsu, and H. J. Liao (1998). Uplift behaviour of cylindrical anchors in sand. Canadian Geotechnical Journal.

Sutherland, H.B., Finlay, T.W., and Fadl, M.O. (1982). Uplift capacity of embedded anchors in sand. In Proceedings of the 3rd International Conference on the Behaviour of Offshore Structures, Cambridge, Mass., Vol. 2, pp. 451-463.

Tagaya, K., Scott, R.F., and Aboshi, H. (1988). Pullout resistance of buried anchor in sand. Soils and Foundations, 28(3): 114-130.

Vesic, A.S. (1971). Breakout resistance of objects embedded in ocean bottom. Journal of the Soil Mechanics and Foundation Engineering Division, ASCE, 97(9): 1183-1205.

White, D. J., and M. D. Bolton (2004). Displacement and strain paths during plane-strain model pile installation in sand. Geotechnique 54(6): 375-397.

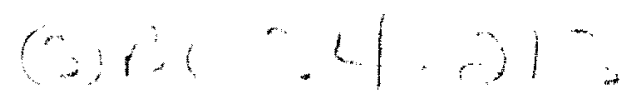

